\title{
A SCALING LIMIT THEOREM FOR A CLASS OF SUPERDIFFUSIONS
}

\author{
BY JÁNOS ENGLÄNDER ${ }^{1}$ AND DMITRY TURAEV \\ EURANDOM and Weierstrass Institute for Applied Analysis and Stochastics
}

Consider the $\sigma$-finite measure-valued diffusion corresponding to the evolution equation $u_{t}=L u+\beta(x) u-f(x, u)$, where

$$
f(x, u)=\alpha(x) u^{2}+\int_{0}^{\infty}\left(e^{-k u}-1+k u\right) n(x, d k)
$$

and $n$ is a smooth kernel satisfying an integrability condition. We assume that $\beta, \alpha \in C^{\eta}\left(\mathbb{R}^{d}\right)$ with $\eta \in(0,1]$, and $\alpha>0$. Under appropriate spectral theoretical assumptions we prove the existence of the random measure

$$
\lim _{t \uparrow \infty} e^{-\lambda_{c} t} X_{t}(d x)
$$

(with respect to the vague topology), where $\lambda_{c}$ is the generalized principal eigenvalue of $L+\beta$ on $\mathbb{R}^{d}$ and it is assumed to be finite and positive, completing a result of Pinsky on the expectation of the rescaled process. Moreover, we prove that this limiting random measure is a nonnegative nondegenerate random multiple of a deterministic measure related to the operator $L+\beta$.

When $\beta$ is bounded from above, $X$ is finite measure-valued. In this case, under an additional assumption on $L+\beta$, we can actually prove the existence of the previous limit with respect to the weak topology.

As a particular case, we show that if $L$ corresponds to a positive recurrent diffusion $Y$ and $\beta$ is a positive constant, then

$$
\lim _{t \uparrow \infty} e^{-\beta t} X_{t}(d x)
$$

exists and equals a nonnegative nondegenerate random multiple of the invariant measure for $Y$.

Taking $L=\frac{1}{2} \Delta$ on $\mathbb{R}$ and replacing $\beta$ by $\delta_{0}$ (super-Brownian motion with a single point source), we prove a similar result with $\lambda_{c}$ replaced by $\frac{1}{2}$ and with the deterministic measure $e^{-|x|} d x$, giving an answer in the affirmative to a problem proposed by Engländer and Fleischmann [Stochastic Process. Appl. 88 (2000) 37-58].

The proofs are based upon two new results on invariant curves of strongly continuous nonlinear semigroups.

Received March 2000.

${ }^{1}$ Supported in part by an EU grant to Stochastic Analysis via Humboldt University, Berlin. AMS 2001 subject classifications. Primary 60J80; secondary 60J60, 60G57.

Key words and phrases. Measure-valued process, superprocess, super-Brownian motion, scaling limit, single point source, invariant curve. 


\section{Introduction and statement of results.}

1.1. Motivation. In [13] it has been proven that the superdiffusion corresponding to the semilinear operator $L u+\beta u-\alpha u^{2}$ tends to a nonzero limit in expectation after exponential rescaling if and only if the linear operator $L+\beta$ satisfies a certain spectral assumption. Although the statement was proved for the case when $\alpha$ and $\beta$ are positive constants, it is easy to check that the proof works just as well in the variable coefficient case. A similar result has been presented in [7] for a nonregular setting (super-Brownian motion with a single point source).

In this paper we replace the expectations by the superdiffusions themselves and prove that the rescaled superdiffusions tend to a limit in law. For the case of the super-Brownian motion with a single point source this will give a positive answer to a problem proposed in [7].

1.2. Preparation. We begin with some notation. Let $\mathcal{M}=\mathcal{M}\left(\mathbb{R}^{d}\right)$ denote the set of finite measures $\mu$ on $\mathbb{R}^{d}$ endowed with the topology of weak convergence and with $\|\mu\|$ denoting the total mass of $\mu$; let $\mathcal{M}_{c}=\mathcal{M}_{c}\left(\mathbb{R}^{d}\right)$ denote the subset of all compactly supported measures. Write $C^{k, \eta}=C^{k, \eta}\left(\mathbb{R}^{d}\right)$ for the usual Hölder spaces of index $\eta \in(0,1]$ including derivatives of order $k$, and set $C^{\eta}:=C^{0, \eta}$. Let $C_{b}=C_{b}\left(\mathbb{R}^{d}\right)$ and $C_{b}^{+}=C_{b}^{+}\left(\mathbb{R}^{d}\right)$ denote the space of bounded continuous functions on $\mathbb{R}^{d}$ and the space of nonnegative bounded continuous functions, respectively; let $\|\cdot\|$ denote the sup-norm for bounded functions. Furthermore, $C=C\left(\mathbb{R}^{d}\right)$ and $C_{0}=C_{0}\left(\mathbb{R}^{d}\right)$ refer to continuous functions on $\mathbb{R}^{d}$ and continuous functions on $\mathbb{R}^{d}$ decaying to zero, respectively. Finally, $C_{c}\left(C_{c}^{+}\right)$denotes the space of continuous (nonnegative continuous) functions on $\mathbb{R}^{d}$ with compact support.

We now recall the definition of $\left(L, \beta, \alpha ; \mathbb{R}^{d}\right)$-superdiffusion. Let $L$ be an elliptic operator on $\mathbb{R}^{d}$ of the form

$$
L=\frac{1}{2} \nabla \cdot a \nabla+b \cdot \nabla \quad \text { on } \mathbb{R}^{d},
$$

where $a_{i j}, b_{i} \in C^{1, \eta}, i, j=1, \ldots, d$, for some $\eta \in(0,1]$ and the symmetric matrix $a=\left\{a_{i j}\right\}$ satisfies

$$
\sum_{i, j=1}^{d} a_{i j}(x) v_{i} v_{j}>0 \quad \text { for all } v \in \mathbb{R}^{d} \backslash\{0\} \text { and all } x \in \mathbb{R}^{d} .
$$

In addition, let $\alpha, \beta \in C^{\eta}$, where $\beta$ is bounded from above (we will later relax this condition) and $\alpha$ is positive.

Notation 1 (Superdiffusion). Let $\left(X, P_{\mu}, \mu \in \mathcal{M}\right)$ denote the $\left(L, \beta, \alpha ; \mathbb{R}^{d}\right)$ superdiffusion. That is, $X$ is the unique $\mathcal{M}$-valued continuous (time-homogeneous) Markov process which satisfies, for any bounded continuous $g: \mathbb{R}^{d} \mapsto \mathbb{R}_{+}$,

$$
E_{\mu} \exp \left\langle X_{t},-g\right\rangle=\exp \langle\mu,-u(\cdot, t)\rangle,
$$


where $u$ is the minimal nonnegative solution to

$$
\begin{gathered}
u_{t}=L u+\beta u-\alpha u^{2} \quad \text { on } \mathbb{R}^{d} \times(0, \infty), \\
\lim _{t \rightarrow 0+} u(\cdot, t)=g(\cdot)
\end{gathered}
$$

(see [8]). Here $\langle v, f\rangle$ denotes the integral $\int_{\mathbb{R}^{d}} v(d x) f(x)$.

Here is an equivalent way of replacing the word minimal in the definition of $u$ in Notation 1 (cf. [8]): $u$ is the nonnegative solution to (4) obtained as a limit of solutions with Dirichlet boundary condition: $u=\lim _{n \rightarrow+\infty} u_{n}$, where $u_{n}(x, t)$ is the solution to (4) for $|x| \leq n$ with $u_{n}(x)=0$ at $|x|=n$.

REMARK 2. We note that this definition will later be extended to a more general class of $\beta$ 's and a more general class of nonlinearities (see the last subsection of this section).

REMARK 3 (Mild equation with linear semigroup). In fact the parabolic semilinear pde under (4) can be rewritten as an integral equation (or mild equation) as follows: $u$ is the unique function which solves

$$
u(\cdot, t)=T_{t} g-\int_{0}^{t} d s T_{t-s}\left(\alpha u^{2}(\cdot, s)\right),
$$

with $\sup _{0 \leq s \leq t}\|u(\cdot, s)\|<\infty$ for all $t>0$. Here $\left\{T_{t}\right\}_{t \geq 0}$ denotes the semigroup corresponding to the operator $L+\beta$ and acting on $C_{b}$. That is, for bounded and continuous $g$,

$$
T_{t} g:=\mathbb{E}_{x}\left[\exp \left(\int_{0}^{t} \beta\left(Y_{s}\right) d s\right) g\left(Y_{t}\right) ; \tau>t\right],
$$

where $Y$ denotes the diffusion corresponding to $L$ on $\mathbb{R}^{d}$ living on $\mathbb{R}^{d} \cup\{\Delta\}$, the one-point compactification of $\mathbb{R}^{d}$ (with expectations $\left\{\mathbb{E}_{x}\right\}_{x \in \mathbb{R}^{d}}$ ), and $\tau$ denotes its lifetime,

$$
\tau:=\inf \left\{t \geq 0 \mid Y_{t} \notin \mathbb{R}^{d}\right\} .
$$

We mention that the mild equation under (5) is usually written in a slightly different form: $\left\{T_{t}\right\}_{t>0}$ is replaced by the semigroup corresponding to the operator $L$ on $\mathbb{R}^{d}$ and the nonlinearity $\alpha u^{2}$ is replaced by $-\beta u+\alpha u^{2}$ [see, e.g., [8], formula (1.3)]. The advantage of that formulation is that the semigroup then describes the spatial motion (the diffusion corresponding to $L$ on $\mathbb{R}^{d}$ ), while the nonlinear term refers to the branching mechanism built in the construction of $X$. In this paper we chose to include $\beta$ in the linear semigroup as in (6) for technical reasons. For example, we do not have to assume that $\beta$ is bounded from below; the semigroup under (6) makes sense whenever $\beta$ is bounded from above. 
REMARK 4 (Formula for expectation). Using the stochastic representation formula for solutions of parabolic pde's [see [11], formula (5.15)] it is easy to show that $u(x, t):=T_{t} g(x)$ is the minimal nonnegative solution for (4) with $\alpha=0$. From this, it is standard to verify that

$$
E_{\delta_{x}}\left\langle X_{t}, g\right\rangle=T_{t} g(x) .
$$

In the sequel we use concepts and facts from the so-called criticality theory of second-order elliptic operators (see [12], Chapter 4) without further reference. The definitions for subcritical, critical and product-critical operators, for the ground state of a critical operator and its adjoint, and for the generalized principal eigenvalue of $L+\beta$ on $\mathbb{R}^{d}$ are presented in Appendix B. The reader should consult that section from time to time, where a review is given on criticality theory.

We will also use the notation $\langle f, g\rangle$ with nonnegative $f$ and $g$ for the (possibly infinite) integral $\int_{\mathbb{R}^{d}} d x f(x) g(x)$. In [13] the following result has been proved (though formally for a somewhat more restricted case-see the note after the theorem).

THEOREM P. Let $\mu \in \mathcal{M}_{c}$ and let $g \in C_{c}^{+}$be not identically zero. Let $\lambda_{c} \in \mathbb{R}$ denote the generalized principal eigenvalue of $L+\beta$ on $\mathbb{R}^{d}$. In the case when $L+\beta-\lambda_{c}$ is critical we denote the corresponding ground state by $\phi$. (The ground state for the formal adjoint of $L+\beta-\lambda_{c}$ will be denoted by $\tilde{\phi}$.) Finally, let $\rho \in \mathbb{R}$ :

(i) $\lim _{t \uparrow \infty} e^{-\rho t} E_{\mu}\left\langle X_{t}, g\right\rangle=0$ if $\rho>\lambda_{c}$, and $\lim _{t \uparrow \infty} e^{-\rho t} E_{\mu}\left\langle X_{t}, g\right\rangle=\infty$ if $\rho<\lambda_{c}$; then

(ii-a) if $L+\beta-\lambda_{c}$ is subcritical or if $L+\beta-\lambda_{c}$ is critical but $\langle\phi, \tilde{\phi}\rangle=\infty$,

$$
\lim _{t \uparrow \infty} e^{-\lambda_{c} t} E_{\mu}\left\langle X_{t}, g\right\rangle=0 ;
$$

(ii-b) if $L+\beta-\lambda_{c}$ is critical and $\langle\phi, \tilde{\phi}\rangle<\infty$, then

$$
\lim _{t \uparrow \infty} e^{-\lambda_{c} t} E_{\mu}\left\langle X_{t}, g\right\rangle=\langle\mu, \phi\rangle\langle\tilde{\phi}, g\rangle,
$$

where $\phi$ and $\tilde{\phi}$ are normalized by $\langle\phi, \tilde{\phi}\rangle=1$.

The condition in (ii-b) of Theorem $\mathrm{P}$ is sometimes called product criticality (see Appendix B for more explanation).

As far as (i) and (ii-a) are concerned, the proofs given in [13] require some further completion. In fact, it is very easy to complete the proof of the first statement in (i) and the proof of (ii-a) under the subcriticality assumption; however, the second statement in (i) and the critical but not product-critical case in (ii-a) are not proven in full generality in that paper. Nevertheless, since in the present article we will use (ii-b) only [except in Remark 5, where we use the first statement in (i)], we defer the discussion regarding (i) and (ii-a) to the forthcoming paper [9]. 
Although (ii-b) was stated for the case when $L$ is a conservative diffusion (i.e., a diffusion having an infinite lifetime) on $\mathbb{R}^{d}$ with a corresponding $C_{0}$-preserving semigroup and $\beta$ and $\alpha$ are positive constants, it is easy to check that its proof never uses these assumptions and consequently it is valid for our general notion of $\left(L, \beta, \alpha ; \mathbb{R}^{d}\right)$-superdiffusion as well. [Note that if $\beta$ is constant, we have $\lambda_{c}=\beta+\lambda_{c}(L)$, where $\lambda_{c}(L)$ denotes the generalized principal eigenvalue of $L$ on $\mathbb{R}^{d}$.]

In a recent paper [7] a nonregular setting, namely a super-Brownian motion with a single point source, has been studied and a result analogous to Theorem $\mathrm{P}$ has been proved for this process. In this case the additional mass production is zero everywhere except at a single point (the origin, say) where the mass production is infinite (in a $\delta$-function sense). In other words, consider the superdiffusion $X^{\text {sin }}$ corresponding to the formal evolution equation

$$
\begin{gathered}
u_{t}=\frac{1}{2} \Delta u+\delta_{0} u-\alpha u^{2} \quad \text { on } \mathbb{R} \times(0, \infty), \\
u(\cdot, 0)=g(\cdot),
\end{gathered}
$$

where $\delta_{0}$ denotes the Dirac $\delta$-function at zero. The precise meaning of the above evolution equation is that $u$ is the unique (nonnegative) solution to the integral equation

$$
\begin{aligned}
u(\cdot, t)= & \int_{-\infty}^{\infty} d y p(t, \cdot, y) g(y)+\int_{0}^{t} d s p(t-s, \cdot, 0) u(0, s) \\
& -\int_{0}^{t} d s \int_{-\infty}^{\infty} d y p(t-s, \cdot, y) \alpha(y) u^{2}(y, s), \quad t>0
\end{aligned}
$$

with $\sup _{0 \leq s \leq t}\|u(\cdot, s)\|<\infty$ for all $t>0$, where $\{p(t, x, y)=p(t, x-y) ; t>0$, $x, y \in \mathbb{R}\}$ denote the Brownian transition densities. $X^{\sin }$ is then determined by its Laplace functional as in (3), but with $u$ from (8). The corresponding expectations will be denoted by $\left\{E_{\mu}^{\sin }, \mu \in \mathcal{M}_{f}\right\}$.

In [7] the following result is proved for $\alpha=1$ (the proof for general $\alpha>0$ is virtually identical to the proof given in [7]):

THEOREM EF. For all bounded continuous $g: \mathbb{R} \mapsto \mathbb{R}_{+}$and $\mu \in \mathcal{M}(\mathbb{R})$,

$$
\lim _{t \uparrow \infty} e^{-t / 2} E_{\mu}^{\sin }\left\langle X_{t}^{\sin }, g\right\rangle=\left\langle e^{-|x|}, \mu\right\rangle\left\langle e^{-|x|}, g\right\rangle .
$$

Note that in this (nonregular) setting, the number $1 / 2$ and the function $x \mapsto$ $e^{-|x|}$ play the role of $\lambda_{c}$ and $\phi(=\tilde{\phi})$. Note also that $\left\langle e^{-2|x|}, 1\right\rangle=1$; that is, $x \mapsto e^{-|x|}$ has already been "normalized."

An obvious but important fact is recorded in the following remark. 
REMARK 5 ("Overscaling"). By Theorem P(i) and the Markov inequality, for the $\left(L, \beta, \alpha ; \mathbb{R}^{d}\right)$-superdiffusion $X$ we have $\lim _{t \uparrow \infty}\left\langle e^{-\rho t} X_{t}, g\right\rangle=0$ in probability if $\rho>\lambda_{c}$, provided $X_{0} \in \mathcal{M}_{c}$. Similarly, using Theorem EF, $\lim _{t \uparrow \infty}\left\langle e^{-\rho t} X_{t}^{\text {sin }}, g\right\rangle=0$ in probability if $\rho>1 / 2$, provided $X_{0} \in \mathcal{M}(\mathbb{R})$.

Motivated by these results and a proposed problem in [7] (see Remark 3 in that paper), we ask the following natural questions. Let the $\left(L, \beta, \alpha ; \mathbb{R}^{d}\right)$-superdiffusion $X$ satisfy the condition in (ii-b) of Theorem P. Does the rescaled process $e^{-\lambda_{c} t} X_{t}$ itselft have a limit in law for any $X_{0} \in \mathcal{M}_{c}$ ? Is the same true for the rescaled process $e^{-t / 2} X_{t}^{\text {sin }}$ for any $X_{0} \in \mathcal{M}(\mathbb{R})$ ?

To answer these questions, we first invoke the definition of local extinction.

DEFINITION 6 (Local extinction). A measure-valued path $X$ exhibits local extinction if $X_{t}(B)=0$ for all sufficiently large $t$, for each ball $B$. The measurevalued process $X$ corresponding to $P_{\mu}$ is said to possess this property if it is true with $P_{\mu}$-probability 1 .

Roughly speaking, local extinction means that the support of the measurevalued process leaves any given compact set in finite time.

REMARK 7 (Process property). In $[8,13]$ it was shown that, for fixed $L, \beta$ and $\alpha$, if the property in Definition 6 holds for some $P_{\mu}, \mu \in \mathcal{M}_{c}$ with $\mu \neq 0$, then it in fact holds for every $P_{\mu}, \mu \in \mathcal{M}_{c}$.

Local extinction can be characterized in terms of $L$ and $\beta$ (see [13], Theorem 6 and Remark 1).

LEMMA 8 (Spectral condition for local extinction). The $\left(L, \beta, \alpha ; \mathbb{R}^{d}\right)$-superdiffusion $X$ exhibits local extinction if and only if there exists a (strictly) positive solution $u$ to the equation $(L+\beta) u=0$ on $\mathbb{R}^{d}$, that is, if and only if $\lambda_{c} \leq 0$.

REMARK 9 (Ergodicity and local extinction). Let $f: \mathbb{R}_{+} \rightarrow \mathbb{R}_{+}$. Using Lemma 8, it immediately follows that if $\lambda_{c} \leq 0$, we have $f(t)\left\langle X_{t}, g\right\rangle \rightarrow 0$ as $t \rightarrow \infty$ a.s. for any $g \in C_{c}^{+}$and $X_{0} \in \mathcal{M}_{c}$, no matter how "large" $f$ is.

Nevertheless, the situation is completely different when replacing $g \in C_{c}^{+}$ by $g \in C_{b}^{+}$. For the case when $\mu \in \mathcal{M}_{c}$ but $g=1$, the condition $\lambda_{c} \leq 0$ (local extinction) does not contain enough information about the behavior of the total mass. To elucidate this point, consider the following example. Fix $\beta, \alpha>0$ and take an $L$ with $\lambda_{c}(L) \leq-\beta$ corresponding to a conservative diffusion. Let $X$ denote the corresponding superdiffusion and let $X^{*}$ denote the superdiffusion where $L$ is replaced by $\frac{1}{2} \Delta$ (supercritical super-Brownian motion). Then $\lambda_{c}\left(\frac{1}{2} \Delta+\beta\right)=\beta$ but for $X$ we have $\lambda_{c}(L+\beta) \leq 0$. Nevertheless, the 
processes $\|X\|$ and $\left\|X^{*}\right\|$ have the same law, because the branching is independent of the motion process and "no mass is lost" due to the conservativeness of the diffusion corresponding to $L$. (See the argument preceding formula (1.4) in [13].) Therefore $\|X\|$ grows exponentially in expectation in this case. On the other hand, the (sub)critical super-Brownian motion exhibits local extinction too but its total mass is constant (resp. tends to zero) in expectation.

Last, we mention that the case when $\lambda_{c} \leq 0$ and $\mu$ does not belong to $\mathcal{M}_{c}$ but rather is $\sigma$-finite has also been studied in the literature. The simplest case is critical super-Brownian motion, that is, $L=\frac{1}{2} \Delta, \beta=0$ and $0<\alpha=$ const. In this case $\lambda_{c}=0$. For the ergodic behavior of this process under different, and even mixed starting measures, see [2]. For $\left(L, \beta, \alpha, \mathbb{R}^{d}\right)$-superdiffusions see [14].

In the sequel we always assume that $\lambda_{c}>0$, that is, that the $\left(L, \beta, \alpha ; \mathbb{R}^{d}\right)$ superdiffusion under consideration does not exhibit local extinction. (As already mentioned in this subsection, in the singular setting the number $1 / 2$ plays the role of $\lambda_{c}$.)

1.3. Scaling limits for superdiffusions. In this paper we prove the existence of the scaling limits in the case of $\left(L, \beta, \alpha ; \mathbb{R}^{d}\right)$-superdiffusions and in the case of the single point source as well, under the assumption that $\lambda_{c}(L+\beta)>0$ and that the condition in (ii-b) of Theorem $\mathrm{P}$ (product criticality) holds. In addition, we assume that $\alpha$ is not "too large." In fact we are able to replace $\mathcal{M}_{c}$ and $\mathcal{M}(\mathbb{R})$ by two families of measures, each satisfying an integrability assumption only. (See Theorems 1 and 2 below.)

As is usual in the analysis of nonlinear phenomena, we use a geometric approach to (5). For a continuous function $u$ define the weighted norm $\|u\|_{\phi^{-1}}=$ $\sup _{x}\left\|u(x) \phi^{-1}(x)\right\|$, where $\phi$ is the ground state of $L+\beta-\lambda_{c}$. Under certain conditions guaranteed by Theorem 1 or 2 below, we prove in Lemma 20 (Section 3 ) the existence of a special smooth curve $u=\psi(\sigma), \sigma \in[0, \infty)$, in the space of nonnegative functions bounded in the norm $\|\cdot\|_{\phi^{-1}}$, such that $\psi(0)=0$ and $\psi^{\prime}(0)=\phi$ and that the curve is invariant under the positive time shift $u(0) \mapsto u(t)$ defined by (5). Thus, the curve emanates from zero and is tangent at zero to the one-dimensional invariant (with respect to the semigroup $\left\{T_{t}\right\}_{t \geq 0}$ ) subspace, spanned by $\phi$. We prove that this curve is uniquely defined by the condition that for any point $u(0)=g=\psi\left(\sigma_{0}\right)$ on the curve we have

$$
u(t)=\psi\left(\sigma_{0} e^{\lambda_{c} t}\right),
$$

where $u(t)$ is the unique nonnegative solution to (5), bounded in the $\|u\|_{\phi^{-1}}$-norm at all $t$. This condition means that the curve is parametrized in such a way that (5) restricted to the invariant curve becomes linear: $\dot{\sigma}=\lambda_{c} \sigma$.

Since our invariant curve $u=\psi(\sigma)$ is defined uniquely by the nonlinear equation (5), it is quite legitimate to formulate the results in terms of the 
function $\psi$, as we do below (note that our proof of existence of the invariant curve in Lemma 20 is constructive and gives an algorithm for the computation of the function $\psi)$. In essence, Theorems 1 and 2 illustrate one of the standard ideas of local nonlinear analysis: the analogy between invariant subspaces of linearized evolution equations and invariant curves of nonlinear equations.

Before stating our main result we introduce additional notation.

NOTATION 10. For $0 \leq g$ measurable, define the following space of measures:

$$
\mathcal{M}^{(g)}:=\left\{\mu \text { is a measure on } \mathbb{R}^{d}:\langle\mu, g\rangle<\infty\right\} .
$$

We now state our main result.

THEOREM 1 [Scaling limit for $\left(L, \beta, \alpha ; \mathbb{R}^{d}\right)$-superdiffusions]. Let $X$ be the $\left(L, \beta, \alpha ; \mathbb{R}^{d}\right)$-superdiffusion with $L, \beta, \alpha$ as in the paragraph preceding Notation 1. Let $0<\lambda_{c}$, where $\lambda_{c}$ denotes the generalized principal eigenvalue of $L+\beta$ on $\mathbb{R}^{d}$. Assume that the condition in (ii-b) of Theorem $\mathrm{P}$ (product criticality) holds. In addition, assume that $\alpha \phi$ is bounded from above.

Then, for any $X_{0}=\mu \in \mathcal{M}^{(\phi)}$, there exists a nonnegative nondegenerate random variable $N_{\mu}$ such that, for all $g \in C_{c}^{+}$,

$$
\lim _{t \uparrow \infty} e^{-\lambda_{c} t}\left\langle X_{t}, g\right\rangle=N_{\mu}\langle\tilde{\phi}, g\rangle \quad \text { in law. }
$$

Moreover, under the normalization $\langle\phi, \tilde{\phi}\rangle=1$, the law of $N_{\mu}$ is determined via its Laplace transform as follows:

$$
\mathbf{E} e^{-\sigma N_{\mu}}=\exp \langle\mu,-\psi(\sigma)\rangle, \quad \sigma>0,
$$

where $\sigma \mapsto \psi(\sigma)$ is the invariant curve defined by (10). Furthermore,

$$
\mathbf{E} N_{\mu}=\langle\mu, \phi\rangle \text {. }
$$

In particular, $\mathbf{P}\left(N_{\mu}<\infty\right)=1$.

If we assume in addition that $\phi$ is bounded away from zero, then

$$
\lim _{t \uparrow \infty} e^{-\lambda_{c} t} X_{t}(d x)=N_{\mu} \tilde{\phi}(x) d x \quad \text { in law. }
$$

REMARK 11 (Joint distribution). The reader can easily check that the following (apparently stronger) "vector formulation" is in fact equivalent to (11): For any $n \in \mathbb{N}$ and any collection of $C_{c}^{+}\left(\mathbb{R}^{d}\right)$-functions $\left(g_{1}, \ldots, g_{n}\right)$,

$$
\lim _{t \rightarrow \infty} e^{-\lambda_{c} t}\left(\left\langle X_{t}, g_{1}\right\rangle, \ldots,\left\langle X_{t}, g_{n}\right\rangle\right)=N_{\mu}\left(\left\langle\tilde{\phi}, g_{1}\right\rangle, \ldots,\left\langle\tilde{\phi}, g_{n}\right\rangle\right) \quad \text { in law. }
$$

An interpretation of Theorem 1 will be given in the next subsection. 
REMARK 12. It is not hard to show that (11) implies that

$$
\mathbf{P}\left(N_{\mu}=0\right) \geq P_{\mu}\left(\left\|X_{t}\right\|=0 \text { for all large } t \text { 's }\right) .
$$

(We defer the proof to the next subsection, because we will need the concept of the $h$-transform for superprocesses defined in that subsection.) The right-handside probability, that is, the probability of finite time extinction, is positive for all $\mu \in \mathcal{M}_{c}$ (see [8], Theorem 3.1), and consequently $\mathbf{P}\left(N_{\mu}=0\right)>0$ for all $\mu \in \mathcal{M}_{c}$.

REMARK 13 (Example for invariant curve). When $\alpha$ and $\beta$ are positive constants and $L, \alpha$ and $\beta$ satisfy the conditions of Theorem 1, a straightforward computation (left to the reader) gives that

$$
\psi(\sigma)=\frac{\beta \sigma}{\beta+\alpha \sigma}, \quad \sigma \geq 0 .
$$

We continue with two proposed problems.

Problem 14. Is it true in general that

$$
\mathbf{P}\left(N_{\mu}=0\right)=P_{\mu}\left(\left\|X_{t}\right\|=0 \text { for all large } t \text { 's }\right) \text { ? }
$$

(Compare [1], Theorem III.7.2, for nonspatial branching processes.)

PROBLEM 15. What can we say about the asymptotic behavior of $X$ in the case when $L+\beta-\lambda_{c}$ is subcritical or $L+\beta-\lambda_{c}$ is critical but $\langle\phi, \tilde{\phi}\rangle=\infty$ [case (ii-a) in Theorem P]?

REMARK 16 (Supercritical super-Brownian motion). The simplest case of Problem 15 is when $X$ corresponds to the quadruple $\left(\frac{1}{2} \Delta, \beta, \alpha ; \mathbb{R}^{d}\right), d \geq 1$, with $\alpha, \beta$ positive constants (supercritical super-Brownian motion). Here $\lambda_{c}=\beta$ and

$$
\frac{1}{2} \Delta+\beta-\lambda_{c}=\frac{1}{2} \Delta \text {. }
$$

Since $\phi=\tilde{\phi} \equiv 1, d \geq 1$, the operator $\frac{1}{2} \Delta$ either is critical but not product-critical $(d \leq 2)$ or is subcritical $(d \geq 3)$.

In the forthcoming paper [9] we will prove that, for $g \in C_{c}^{+}$,

$$
\lim _{t \uparrow \infty}(2 \pi t)^{d / 2} e^{-\beta t}\left\langle X_{t}, g\right\rangle=Z_{1}\langle d x, g\rangle \quad \text { in law, }
$$

where $Z$ is the total mass process of the critical super-Brownian motion corresponding to $\left(\frac{1}{2} \Delta, 0, \alpha / \beta ; \mathbb{R}^{d}\right)$ and $Z$ starts with $\left\|X_{0}\right\| . Z$ is also known as Feller's diffusion. In fact (see, e.g., [13], page 239) $Z$ is the diffusion process corresponding to the operator $\frac{\alpha}{\beta} x \frac{d^{2}}{d x^{2}}$ on $[0, \infty)$, where 0 is a cemetery state [or, equivalently, it is the unique strong solution of the stochastic differential equation:

$$
d Z_{t}=\sqrt{2 \frac{\alpha}{\beta} Z_{t}} d w_{t}, \quad Z_{0}=\left\|X_{0}\right\|,
$$


where $w=\left(w_{t}\right)_{t \geq 0}$ is the Brownian motion on the real line]. In [9] we will also improve and generalize the main result of the present article.

Finally, we state a theorem analogous to Theorem 1 for the superdiffusion $X^{\text {sin }}$ of Theorem EF (super-Brownian motion with an additional single point source).

THEOREM 2 (Scaling limit in the case of a single point source). Let $X^{\text {sin }}$ be the superdiffusion corresponding to the integral equation (8), and assume that $\alpha(x) \leq K e^{|x|}, K>0$. For any $X(0)=\mu \in \mathcal{M}^{(\exp -|x|)}$, there exists a nonnegative nondegenerate random variable $N_{\mu}$ with $\mathbf{E} N_{\mu}=\left\langle\mu, e^{-|x|}\right\rangle$ satisfying that

$$
\lim _{t \uparrow \infty} e^{-t / 2} X_{t}^{\sin }(d x)=N_{\mu} e^{-|x|} d x \quad \text { in law. }
$$

Furthermore, the law of $N_{\mu}$ is determined via its Laplace transform as in (12), where $\sigma \mapsto \psi(\sigma)$ is the invariant curve defined by (10) when replacing the nonlinear equation (5) with (8), and using the formal substitution $\lambda_{c}=1 / 2$.

1.4. An interpretation of our main theorem via reducing it to a particular case. Before presenting an interpretation of Theorem 1, first recall the definition of the $h$-transformed superdiffusion. (The $h$-transform for $\left(L, \beta, \alpha ; \mathbb{R}^{d}\right)$-superdiffusions was developed in [8].)

DEFINITION 17 ( $h$-Transformed superdiffusion $X^{h}$ ). Let $0<h \in C^{2, \eta}$ and consider the $\left(L, \beta, \alpha ; \mathbb{R}^{d}\right)$-superdiffusion $X$. Define

$$
X_{t}^{h}:=h X_{t} \quad\left(\text { i.e., } \frac{d X_{t}^{h}}{d X_{t}}=h\right), \quad t \geq 0 .
$$

Then $X^{h}$ is the $\left(L_{0}^{h}, \beta^{h}, \alpha^{h} ; \mathbb{R}^{d}\right)$-superdiffusion, where

$$
L_{0}^{h}:=L+a \frac{\nabla h}{h} \cdot \nabla, \quad \beta^{h}:=\frac{(L+\beta) h}{h} \text { and } \quad \alpha^{h}:=\alpha h .
$$

$X^{h}$ makes sense even if $\beta^{h}$ is unbounded from above (see [8], Section 2, for more elaboration). $X^{h}$ is called the $h$-transformed superdiffusion.

REMARK 18 ( $h$-Transforms). (i) $L_{0}^{h}$ is just the diffusion part of the usual linear $h$-transformed operator $L^{h}$ (see [12], Chapter 4).

(ii) The operators $\mathcal{A}(u):=L u+\beta u-\alpha u^{2}$ and $\mathcal{A}^{h}(u):=L_{0}^{h} u+\beta^{h} u-\alpha^{h} u^{2}$ are related by $\mathcal{A}^{h}(u)=\frac{1}{h} \mathcal{A}(h u)$.

REMARK 19 (Invariance under $h$-transforms). An obvious but important property of the $h$-transform is that it leaves invariant the support process $t \mapsto$ $\operatorname{supp}\left(X_{t}\right)$ of $X$. 
We now give an interpretation of Theorem 1 using the transformed process $X^{\phi}=\phi X$ as follows. First note that $\phi$ and $\tilde{\phi}$ transform into 1 and $\phi \tilde{\phi}$, respectively. Hence, Theorem 1 states that, for $X_{0}^{\phi}=v \in \mathcal{M}$,

$$
\lim _{t \uparrow \infty} e^{-\lambda_{c} t} X_{t}^{\phi}(d x)=N_{\nu}^{\phi} \phi \tilde{\phi} d x \quad \text { in law }
$$

(cf. [1], Theorem III.7.1, for nonspatial branching processes). Recall that $X^{\phi}$ is the $\left(L_{0}^{\phi}, \lambda_{c}, \alpha \phi ; \mathbb{R}^{d}\right)$-superdiffusion. (Note that $\beta^{\phi}=\lambda_{c}$ is no longer spatially dependent.)

Next, note that integrating against the function 1 in (18) yields

$$
\lim _{t \uparrow \infty} e^{-\lambda_{c} t}\left\|X_{t}^{\phi}\right\|=N_{\nu}^{\phi} \quad \text { in law; }
$$

that is, the total mass behaves like $e^{\lambda_{c} t} N_{v}^{\phi}$ as $t \rightarrow \infty$. Recall that $\lambda_{c}$ is the average mass creation at each point of $\mathbb{R}^{d}$ and note that, since $\phi$ transforms into 1 , we have

$$
\mathbf{E} N_{v}^{\phi}=\|v\| \text {. }
$$

By (12) (applied for the $\phi$-transformed setting) $N_{v}^{\phi}$ depends on the whole branching term $\lambda_{c} u-\alpha \phi u^{2}$, where $\alpha \phi$ can be identified with the variance of the offspring distribution (see [8], Appendix 1). It depends also on $L_{0}^{\phi}$, that is, on the motion process, which fact comes of course from the spatial dependence of the branching.

Note also (see Appendix B) that by the product-criticality assumption, and by the invariance of this property under $h$-transforms, $L_{0}^{\phi}$ corresponds to a positive recurrent diffusion (loosely speaking, positive recurrence means that the diffusion hits any fixed ball in finite expected time) which ergodizes with invariant measure $\phi \tilde{\phi} d x$ (see [12], Theorem 4.9.9). Putting this together with (19), the right-hand side of the approximating formula

$$
X_{t}^{\phi}(d x) \sim e^{\lambda_{c} t} N_{v}^{\phi} \phi \tilde{\phi} d x
$$

can be interpreted as $e^{\lambda_{c} t} N_{\nu}^{\phi}$ being the total mass and $\phi \tilde{\phi} d x$ being the limiting distribution of the individual particle.

We close this section by proving Remark 12 .

PROOF OF REMARK 12. It is enough to prove the inequality for $X^{\phi}$, because the probability of extinction is the same for $X$ (starting with $\mu$ ) and $X^{\phi}$ (starting with $v=\phi \mu)$, and also $\mathbf{P}\left(N_{\mu}=0\right)=\mathbf{P}\left(N_{\nu}^{\phi}=0\right)$. Using (18), we have

$$
\begin{aligned}
\mathbf{P}\left(N_{v}^{\phi}=0\right) & =\lim _{s \uparrow \infty} \mathbf{E} e^{-s N_{v}^{\phi}\langle\phi \tilde{\phi}, 1\rangle}=\lim _{s \uparrow \infty t \uparrow \infty} \lim _{t \uparrow} \mathbf{E} e^{-s\left\langle e^{-\lambda_{c} t} X_{t}^{\phi}, 1\right\rangle} \\
& \geq P_{\mu}\left(\left\langle X_{t}^{\phi}, 1\right\rangle=0 \text { for all large } t \text { 's }\right) .
\end{aligned}
$$

This completes the proof of the remark. 
1.5. More general branching. In this subsection we consider superdiffusions with more general branching mechanisms and generalize our main theorem for that setup. To this end, first recall that in [8] the definition of $\left(L, \beta, \alpha ; \mathbb{R}^{d}\right)$-superdiffusion was extended for $\beta$ 's which are not necessarily bounded from above but rather satisfy the more general condition

$$
\lambda_{c}=\lambda_{c}(L+\beta)<\infty .
$$

This extension relies on the fact that the $h$-transform with $h=\phi$ transforms formally the quadruple $\left(L, \beta, \alpha ; \mathbb{R}^{d}\right)$ into the quadruple $\left(L_{0}^{\phi}, \lambda_{c}, \alpha \phi ; \mathbb{R}^{d}\right)$, which corresponds to a superdiffusion $X$ (since $\left.\beta^{h}=\lambda_{c}<\infty\right)$. Then the $\left(L, \beta, \alpha ; \mathbb{R}^{d}\right)$ superdiffusion $\hat{X}$ can be defined by $\hat{X}:=\frac{1}{\phi} X$ (where $X$ starts at $X_{0}=\mu \in \mathcal{M}_{c}$ if and only if $\hat{X}$ starts at $\left.\hat{X}_{0}=\frac{1}{\phi} \mu \in \mathcal{M}_{c}\right) . \hat{X}$, however, is not $\mathcal{M}$-valued in general but rather $\sigma$-finite measure-valued. (See [8] for more elaboration.) In particular, the appropriate topology for measures becomes the vague topology in place of the weak one.

In fact, this construction can easily be generalized for (time-independent) local branching, that is, for the case when instead of the quadratic nonlinearity in (5) we have the more general nonlinearity of the form

$$
f(x, u)=\alpha(x) u^{2}(x)+\int_{0}^{\infty}\left[e^{-k u(x)}-1+k u(x)\right] n(x, d k) .
$$

Here $n$ is a kernel from $\mathbb{R}^{d}$ to $[0, \infty)$, that is, $n(x, d k)$ is a measure on $[0, \infty)$ for each $x \in \mathbb{R}^{d}$, and $n(\cdot, B)$ is a continuous function on $\mathbb{R}^{d}$ for every measurable $B \subseteq[0, \infty$ ) (cf. [5], Sections 1.7-1.8). (In the original setting of [5] only the measurability was required. We, however, prefer to work in this paper with the spaces of continuous functions.) To be able to define the superdiffusion $\hat{X}$ corresponding to $L, \beta$ and $f$ via an $h$-transform, we assume that $0<\alpha \phi$ is bounded from above and that $n$ satisfies

$$
\sup _{x \in \mathbb{R}^{d}} \int_{0}^{\infty}\left[k \wedge \phi(x) k^{2}\right] n(x, d k)<\infty .
$$

Moreover, we assume that the convergence to the limit

$$
\lim _{K \rightarrow+\infty} \int_{K}^{\infty} k n(x, d k)=0
$$

is uniform with respect to $x$ on every compact subset of $\mathbb{R}^{d}$. [This condition will guarantee that the map $x \mapsto f(x, u(x))$ is continuous whenever $u \in C$.]

The $h$-transform with $h=\phi$ takes the operator $L+\beta$ into $L_{0}^{\phi}+\lambda_{c}$, while $f(x, u)$ transforms into

$$
f^{\phi}(x, u)=\alpha \phi(x) u^{2}(x)+\int_{0}^{\infty}\left[e^{-k u(x)}-1+k u(x)\right] n^{\phi}(x, d k),
$$


where

$$
n^{\phi}(x, d k):=\frac{1}{\phi(x)} n\left(x, \frac{d k}{\phi(x)}\right) .
$$

Note that, by (22), $n^{\phi}$ satisfies

$$
\sup _{x \in \mathbb{R}^{d}} \int_{0}^{\infty}\left(k \wedge k^{2}\right) n^{\phi}(x, d k)<\infty
$$

(and this integral converges uniformly with respect to $x$ ). Using this, along with the fact that $\alpha \phi$ is bounded from above by assumption, the $\phi$-transformed mild equation uniquely defines a superdiffusion $X$ (see [5], Sections 1.6-1.8). Then the superdiffusion $\hat{X}$ can be defined in the usual way: $\hat{X}:=\frac{1}{\phi} X$. (Note that $\hat{X}$ is $\mathcal{M}^{\phi}$-valued for every starting measure in $\mathcal{M}^{\phi}$. In particular, if $\phi$ is bounded away from zero, then $\hat{X}$ is $\mathcal{M}$-valued for every starting measure in $\mathcal{M}^{\phi}$.) Denote the semigroup corresponding to $L_{0}^{\phi}+\lambda_{c}$ by $\left\{T_{t}^{\phi}\right\}_{t \geq 0}$. It is immediately seen that $\hat{X}$ corresponds to the mild equation

$$
u(\cdot, t)=T_{t} g-\int_{0}^{t} d s T_{t-s}(f(u(\cdot, s))),
$$

where the linear semigroup $\left\{T_{t}\right\}_{t \geq 0}$ is defined by

$$
T_{t}(u):=\phi T_{t}^{\phi}(u / \phi), \quad t \geq 0,
$$

and the nonlinearity $f$ is defined by

$$
f(x, u):=\phi(x) f^{\phi}(x, u / \phi) .
$$

(The $h$-transformed mild equation is defined whenever the initial function at $t=0$ belongs to $C_{c}^{+}-$see [8] for further explanation for the case when $n \equiv 0$.)

In fact, Theorem $\mathrm{P}$ and the remark preceding it are still true for this more general setup. Our proof of Theorem 1 still works for this more general setup if [in addition to (20), the boundedness of $\alpha \phi$ and the product-criticality assumption of the theorem] one requires that

$$
\sup _{x \in \mathbb{R}^{d}} \int_{0}^{\infty}\left[\phi^{\delta}(x) k^{1+\delta} \wedge \phi^{2}(x) k^{2}\right] n(x, d k)<\infty \quad \text { for some } \delta>0 .
$$

This will guarantee that the Hölder-type condition (32) in Lemma 25 is satisfied for the nonlinearity $f^{\phi}$. Then Lemma 25 yields the existence of a unique smooth invariant curve defined by (10) for the nonlinear equation (25).

We summarize the above in a proposition. Let us call the superdiffusion described in this section the $\left(L, \beta, f ; \mathbb{R}^{d}\right)$-superdiffusion. (The term "superdiffusion" might suggest that the process has continuous trajectories; in fact, in the general case, when $f$ is not merely quadratic, $X$ can have "jumps".) 
Proposition 20 [Scaling limit for $\left(L, \beta, f ; \mathbb{R}^{d}\right)$-superdiffusions]. Let $X$ be the $\left(L, \beta, f ; \mathbb{R}^{d}\right)$-superdiffusion with $L$ as in the paragraph preceding Notation 1 and the nonlinearity $f(x, u)$ given by (21) where (26) is satisfied. Let $0<\lambda_{c}<\infty$, where $\lambda_{c}$ denotes the generalized principal eigenvalue of $L+\beta$ on $\mathbb{R}^{d}$. Assume that the condition in (ii-b) of Theorem $P$ (product criticality) holds. In addition, assume that $\alpha \phi$ is bounded from above.

Then, for any $X_{0}=\mu \in \mathcal{M}^{(\phi)}$, there exists a nonnegative nondegenerate random variable $N_{\mu}$ such that

$$
\lim _{t \uparrow \infty} e^{-\lambda_{c} t} X_{t}(d x)=N_{\mu} \tilde{\phi}(x) d x \quad \text { in law. }
$$

(Here the convergence is with respect to the vague topology.)

Moreover, under the normalization $\langle\phi, \tilde{\phi}\rangle=1$, the law of $N_{\mu}$ is determined via its Laplace transform as follows:

$$
\mathbf{E} e^{-\sigma N_{\mu}}=\exp \langle\mu,-\psi(\sigma)\rangle \sigma>0,
$$

where $\sigma \mapsto \psi(\sigma)$ is the invariant curve defined by (10) for the nonlinear equation (25).

Furthermore,

$$
\mathbf{E} N_{\mu}=\langle\mu, \phi\rangle,
$$

and in particular, $\mathbf{P}\left(N_{\mu}<\infty\right)=1$.

If we assume in addition that $\phi$ is bounded away from zero, then $X$ is $\mathcal{M}$-valued and (27) holds with respect to the weak topology.

Letting $\alpha \equiv 0$ and choosing an appropriate $n$ (see [5], Section 1.8), (21) has the form

$$
f(x, u)=c(x) u^{1+p}, \quad 0<p<1,
$$

with some nonnegative, nonzero continuous function $c$. In this case (23) and (26) will be satisfied (with $\delta=p$ ) if we assume that $c \phi^{p}$ is bounded from above. (Alternatively, one can slightly modify the proof of Theorem 1 by writing $u^{1+p}$ in place of $u^{2}$ everywhere. Since $f$ transforms into $c \phi^{p} u^{1+p}$ under an $h$-transform with $h=\phi$, the proof goes through when assuming the boundedness of $c \phi^{p}$.)

1.6. Outline. In Section 2 we present examples for Theorem 1. In Section 3 we state and prove two lemmas on invariant curves which play a key role in the proofs. In Section 4 some preparations are made before turning to the proofs, and we also state Theorem 3, an auxiliary result on the recurrence of diffusion processes which we will use in the proof of our main theorem and which may be of independent interest. Section 5 is devoted to the proofs of Theorems 1 and 2 and of Proposition 20. Appendix A presents the proof of Theorem 3. Finally, Appendix B collects some known auxiliary material on the criticality theory of second-order elliptic operators. 
2. Examples. In this section we present applications of our main result for three families of superdiffusions. In the first two examples the underlying motion process (corresponding to the operator $L$ ) is recurrent; in the last example, it is transient.

Our first example has actually been discussed in Section 1.4. In fact, as we have seen, every superdiffusion $X$ satisfying the conditions of Theorem 1 can be $h$-transformed (with $h=\phi$ ) into the type of superdiffusion of the following example.

EXAMPLE 21 (Positive recurrent motion process, $0<\beta=$ const). Let $L$ correspond to a positive recurrent diffusion and let $0<\beta=$ const. Finally, let $\alpha$ be bounded from above. Then $L+\beta-\lambda_{c}=L$, because $\lambda_{c}(L)=0$ by the recurrence property; and $\phi=1$. Furthermore, since the diffusion process is positive recurrent, the operator $L$ is product-critical (i.e., $\tilde{\phi} \in L^{1}$ ). Therefore, (14) holds for any finite starting measure with $\lambda_{c}=\beta$.

To give a concrete example for a positive recurrent diffusion, let $L$ correspond to an Ornstein-Uhlenbeck process,

$$
L=\frac{1}{2} \Delta-k x \cdot \nabla \quad \text { on } \mathbb{R}^{d}, d \geq 1,
$$

where $k>0$. [It is easy to see (cf. [13], Example 3, page 248) that $\tilde{\phi}(x)=$ $\left(\frac{k}{\pi}\right)^{d / 2} \exp \left(-k|x|^{2}\right)$.]

The next example can be considered as a smooth version of our Theorem 2 . (Recall that formally $\lambda_{c}=1 / 2$ in that theorem.)

EXAMPLE 22 (Super-Brownian motion with compactly supported $\beta$ ). Let $L=\frac{1}{2} \Delta$ on $\mathbb{R}^{d}, d \leq 2$. Let $\beta \in C_{c}^{+}$be not identically zero. By the recurrence of the one- and two-dimensional Brownian motions and by Theorem 4.6.3 in [13], we have $\lambda_{c}>0$. The criticality of $L-\lambda_{c}$ follows by the recurrence of the Brownian motion and by Theorem 4.6.7 in [13]. We now prove that $\phi \in L^{2}\left(\mathbb{R}^{d}\right)$ (product criticality). To see this, first let $d=1$. Note that $\phi$ satisfies $\left(\frac{1}{2} \Delta-\lambda_{c}\right) \phi=0$ outside a compact set and therefore $\phi(x)=$ const $\cdot \exp \left( \pm \sqrt{2 \lambda_{c}} x\right)$ for large $|x|$. By the socalled minimal growth property at infinity (see [13], Theorem 7.3.8) it follows that in fact $\phi(x)=$ const $\cdot \exp \left(-\sqrt{2 \lambda_{c}}|x|\right)$ for large $|x|$. The proof for $d=2$ is similar: using polar coordinates, it is easy to check that $f(x):=\exp \left(-\sqrt{2 \lambda_{c}}|x|\right)$ satisfies $\left(\frac{1}{2} \Delta-\lambda_{c}\right) f \leq 0$ outside a compact set. Putting this together with the fact that $\phi$ satisfies $\left(\frac{1}{2} \Delta-\lambda_{c}\right) \phi=0$ outside a compact set and the minimal growth property of $\phi$ at infinity, we have that $\phi \leq K f$ for $K$ large enough. Therefore, for both $d=1$ and $d=2,(11)$ holds in the present case, provided

$$
\alpha(x) \leq K \exp \left(\sqrt{2 \lambda_{c}}|x|\right), \quad K>0,
$$

and the starting measure $\mu=X_{0}$ satisfies $\left\langle\mu, \exp \left(-\sqrt{2 \lambda_{c}}|x|\right)\right\rangle<\infty$. 
Last, we present an example where $L$ corresponds to a transient diffusion process on $\mathbb{R}^{d}$.

EXAMPLE 23. Let

$$
L=\frac{1}{2} \Delta+k x \cdot \nabla \quad \text { on } \mathbb{R}^{d}, d \geq 1,
$$

where $k>0$. (Note that the diffusion corresponding to $L$ is transient.) Let $\beta$ be a constant satisfying $\beta>k d$. It is easy to see (cf. [13], Example 2, pages 247 and 266) that $\lambda_{c}=\beta-k d$ and that $L+\beta-\lambda_{c}=L+k d$ is product-critical with $\phi(x)=\exp \left(-k|x|^{2} / 2\right)$ and $\tilde{\phi}(x)=1$. Therefore, (11) holds with $\lambda_{c}=\beta-k d$, whenever the starting measure $\mu=X_{0}$ satisfies $\left\langle\mu, \exp \left(-k|x|^{2} / 2\right)\right\rangle<\infty$ and

$$
\alpha(x) \leq K \exp \left(k|x|^{2} / 2\right), \quad K>0 .
$$

Note that, if $\beta \leq k d$, the superdiffusion $X_{t}$ exhibits local extinction for any $\mu \in \mathcal{M}_{c}$.

3. Two results concerning invariant curves. Let $\mathcal{X}$ be a Banach space and let $\left\{T_{t}\right\}_{t \geq 0}$ be a strongly continuous semigroup of bounded linear operators acting on $\mathcal{X}$. Let $\mathcal{X}^{+} \subseteq \mathcal{X}$ be a cone. Consider the equation

$$
u(t)=T_{t} u(0)+\int_{0}^{t} T_{t-s} \circ f(u(s)) d s,
$$

for which we assume that it defines for any $u(0) \in \mathcal{X}^{+}$its semiorbit-a curve $u(t)$, $t \geq 0$ in $\mathcal{X}^{+}$. We assume that $f: \mathcal{X} \rightarrow \mathcal{X}$ is smooth; that is, it is differentiable and its derivative is bounded and uniformly continuous on bounded subsets of $\mathcal{X}$. It is easy to see in this case that the semiorbit $u(t)$ defined by (30) is continuous with respect to $t$ and is smooth with respect to the initial condition $u(0)$.

We also assume that

$$
f(0)=0, \quad f^{\prime}(0)=0
$$

and that for the derivative map $F(u): d u \rightarrow f^{\prime}(u) d u$ we have

$$
\|F(u)\| \leq K\|u\|^{\delta}
$$

(in the usual operator-norm) for some positive constants $K$ and $\delta$ and all small $u$. It follows, in particular, that

$$
\|f(u)\| \leq K\|u\|^{1+\delta} .
$$

Concerning the linear semigroup $T_{t}$, we assume that it has an eigenvector $\phi$,

$$
T_{t} \phi=e^{\lambda t} \phi,
$$

for some $\lambda>0$, and that $\phi \in \operatorname{int}\left(\mathcal{X}^{+}\right)$[here $\operatorname{int}\left(\mathcal{X}^{+}\right)$denotes the interior of the cone $\mathcal{X}^{+}$in norm-topology]. Since the vector $\phi$ is defined only modulo a scalar factor, we normalize it by $\|\phi\|=1$. We also assume that, for some constant $M>0$,

$$
\left\|T_{t}\right\| \leq M e^{(\lambda+\varepsilon) t},
$$


where (and this is a crucial assumption)

$$
\varepsilon<\lambda \delta,
$$

and $\delta$ is the exponent in the Hölder-type estimate (32).

Definition 24. A curve $\mathcal{Q}$ in $\mathcal{X}$ is called invariant with respect to the system (30) if for any point $u(0)$ on $\mathcal{Q}$ its positive semiorbit $u(t)$ lies in $\mathcal{Q}$.

LEMMA 25 (Existence of a particular invariant curve). Under(31)-(36), there exists a unique smooth invariant curve $\mathcal{Q}$ lying in $\mathcal{X}^{+}$, parametrically written as $u=\psi(\sigma), \sigma \in[0, \infty)$, where $\psi(0)=0, \psi^{\prime}(0)=\phi$ (i.e., $\mathcal{Q}$ starts at zero and it is tangent at zero to the eigenvector $\phi$ of the linear semigroup), such that for any $\sigma_{0}$, for the point $u(0)=\psi\left(\sigma_{0}\right)$ on $\mathcal{Q}$, its semiorbit is given by

$$
u(t)=\psi\left(e^{\lambda t} \sigma_{0}\right)
$$

REMARK 26. Note that for any point $u(0)=\psi\left(\sigma_{0}\right)$ on $\mathcal{Q}$ there exists a negative semiorbit defined [just by (37)] for any $t \leq 0$, such that it tends to zero and is tangent at zero to $\phi$ as $t \rightarrow-\infty$.

REMARK 27. Note that we parametrize the curve $\mathcal{Q}$ in such a way that the system becomes linear on $\mathbb{Q}: \dot{\sigma}=\lambda \sigma$.

REMARK 28. Although our proof is more or less standard (see [15] for a comparison), our invariant curve result itself is not a standard one because we do not require the usual spectral gap assumption [recall that $\varepsilon \geq 0$ in (35)]. Note that an analogous statement (in a different setting) can be found in [6].

PROOF OF LEMma 25. It is enough to define the function $\psi$ at small $\sigma$ only and show that $\psi(\sigma)$ lies in $\mathcal{X}^{+}$for small $\sigma$ 's: given any point $u(0)=\psi\left(\sigma_{0}\right)$ on the curve $\mathcal{Q}$ with an arbitrarily small $\sigma_{0}$ the function $\psi$ is defined at all larger $\sigma$ by (37), because the positive semiorbit $u(t)$ of $u(0)$ is defined at all $t \geq 0$ by assumption.

So, take any sufficiently small $\sigma$ and consider the equation

$$
v(t)=\sigma \phi+e^{-\lambda t} \int_{-\infty}^{t} T_{t-s} \circ f\left(e^{\lambda s} v(s)\right) d s,
$$

where $t \leq 0$. Here, the unknown is a bounded continuous function $v:[-\infty, 0]$ $\rightarrow \mathcal{X}$. We will find it as a fixed point of the operator $v \mapsto \bar{v}$ defined by

$$
\bar{v}(t)=\sigma \phi+e^{-\lambda t} \int_{-\infty}^{t} T_{t-s} \circ f\left(e^{\lambda s} v(s)\right) d s, \quad t \in[-\infty, 0] .
$$

Conditions (31)-(36) imply (see below) that for all sufficiently small $\sigma$ it is a smooth, contracting operator which maps the set $V$ of continuous functions $v(t)$ 
bounded, say, as $\|v(t)\| \leq 2|\sigma|$, into $V$ itself. Therefore, by the Banach principle of contraction mappings, it has a uniquely defined fixed point in $V$, which depends on $\sigma$ smoothly. Equivalently, (38) has a unique solution $v^{*}$ for all small $\sigma$ which is uniformly bounded for all $t \leq 0$ :

$$
\sup _{t \leq 0}\left\|v^{*}(t ; \sigma)\right\| \leq 2|\sigma| .
$$

Note that $v \equiv 0$ solves (38) at $\sigma=0$. Hence, by uniqueness,

$$
v^{*}(t ; 0) \equiv 0 \text {. }
$$

Since $v^{*}(t ; \sigma)$ is a fixed point of a smooth contracting operator, its derivative $\frac{\partial}{\partial \sigma} v^{*}$ is found as the unique solution of the equation obtained by the formal differentiation of (38):

$$
\frac{\partial}{\partial \sigma} v(t)=\phi+\int_{-\infty}^{t} T_{t-s} \circ e^{\lambda s} f^{\prime}\left(e^{\lambda s} v^{*}(s ; \sigma)\right) \frac{\partial}{\partial \sigma} v(s) d s .
$$

By (41) and (42) we immediately have

$$
\frac{\partial}{\partial \sigma} v^{*}(t ; 0) \equiv \phi
$$

Now we define the function $u^{*}(t ; \sigma) \equiv e^{\lambda t} v^{*}(t ; \sigma)$. By uniqueness of $v^{*}$, the function $u^{*}$ is defined as the unique (bounded by $2|\sigma| e^{\lambda t}$ ) solution of

$$
u(t)=\sigma e^{\lambda t} \phi+\int_{-\infty}^{t} T_{t-s} \circ f(u(s)) d s
$$

[compare this with (38)]. Recall that we define the function $v^{*}$ at nonpositive $t$ only, so the function $u^{*}$ is, by now, defined only at $t \leq 0$ as well. We define $u^{*}(t ; \sigma)$ at $t \geq 0$ as the positive semiorbit of the point $u^{*}(0 ; \sigma)$ defined by the system (30). Comparing (30) and (44) shows that the function $u^{*}$ satisfies (44) at all $t$ (we take into account that $T_{t} \phi=e^{\lambda t} \phi$ by assumption).

Now take any $\tau>0$ and consider the function $u^{* *}(t ; \sigma)=u^{*}\left(t+\tau ; e^{-\lambda \tau} \sigma\right)$. It is immediately seen that, once $u^{*}$ satisfies (44), the function $u^{* *}$ satisfies (44) as well. Therefore, by uniqueness, $u^{* *} \equiv u^{*}$ at all nonpositive $t$ and, in particular,

$$
u^{*}(0, \sigma) \equiv u^{*}\left(\tau ; e^{-\lambda \tau} \sigma\right)
$$

for any $\tau \geq 0$. By definition, this means that the time $\tau$ shift [by the semiflow defined by (30)] of the point $u^{*}\left(0 ; e^{-\lambda \tau} \sigma\right)$ is the point $u^{*}(0, \sigma)$. Thus, if we define the sought function $\psi$ as $\psi(\sigma)=u^{*}(0, \sigma)\left[\equiv v^{*}(0, \sigma)\right]$, we will have that the smooth curve $u=\psi(\sigma)$ is invariant with respect to system (30) and satisfies (37).

Note also that $\psi(0)=0$ and $\psi^{\prime}(0)=\phi$, according to (41) and (43). Thus, this invariant curve will indeed be tangent at zero to the eigenvector $\phi$. Since $\phi \in \operatorname{int}\left(\mathcal{X}^{+}\right)$by assumption, it also follows that $\psi(\sigma)$ lies in $\mathcal{X}^{+}$for all small $\sigma$ 's. 
To show the uniqueness of the curve $Q: u=\psi(\sigma)$ satisfying (37) and $\psi^{\prime}(0)=\phi$, note that if we take any point $u(0)$ on $\mathcal{Q}$ and consider its negative semiorbit $u(t)_{t \leq 0}$ defined by (37), then $u(t)$ must satisfy (44), whose solution is unique as we just have shown [the required boundedness of $u(t)$ by $2 \sigma e^{\lambda} t$ follows from (37) due to the assumed boundedness of $\left.\psi^{\prime}(0)\right]$.

To complete the proof it remains to check that the operator (39) is smooth and contracting on the set $V:\left\{v(s)_{s \in(-\infty, 0]},\|v(s)\| \leq 2|\sigma|\right\}$ and maps this set into itself. First, note that, in (39),

$$
\|\bar{v}(t)\| \leq|\sigma|+\int_{-\infty}^{t}\left\|T_{t-s}\right\| e^{-\lambda t}\left\|f\left(e^{\lambda s} v(s)\right)\right\| d s
$$

(recall that $\|\phi\|=1$ ) and, by virtue of (33) and (35),

$$
\|\bar{v}(t)\| \leq|\sigma|+M K e^{\varepsilon t} \int_{-\infty}^{t} e^{(\lambda \delta-\varepsilon) s}\|v(s)\|^{1+\delta} d s .
$$

Hence,

$$
\sup _{t \leq 0}\|\bar{v}(t)\| \leq|\sigma|+\frac{M K}{\lambda \delta-\varepsilon}\left(\sup _{s \leq 0}\|v(s)\|\right)^{1+\delta}
$$

(recall that $\varepsilon<\lambda \delta$ by assumption). It is clear from this estimate that, for all $\sigma$ small enough, if $\sup _{s \leq 0}\|v(s)\| \leq 2|\sigma|$, then $\|\bar{v}(t)\| \leq 2|\sigma|$ at all $t \leq 0$, which means that the operator under consideration indeed maps the set $V$ into itself.

The smoothness of this operator with respect to $\sigma$ is obvious. To prove the smoothness with respect to $v$ we must check that the linear operator

$$
\Delta v(t) \longmapsto \int_{-\infty}^{t} T_{t-s} e^{-\lambda(t-s)} f^{\prime}\left(e^{\lambda s} v(s)\right) \Delta v(s) d s
$$

obtained by formal differentiation of (39) is well defined and bounded on the space of uniformly bounded $\Delta v(s)_{s \in(-\infty, 0]}$, provided $v(s) \in V$. This is straightforward. In fact, by (35) and (32), we obtain that

$$
\begin{gathered}
\left\|\int_{-\infty}^{t} T_{t-s} e^{-\lambda(t-s)} f^{\prime}\left(e^{\lambda s} v(s)\right) \Delta v(s) d s\right\| \\
\quad \leq M \int_{-\infty}^{t} e^{\varepsilon(t-s)} K(2|\sigma|)^{\delta} e^{\lambda \delta s}\|\Delta v(s)\| d s \\
\quad \leq \frac{M K}{\lambda \delta-\varepsilon}(2|\sigma|)^{\delta} \sup _{s \in(-\infty, 0]}\|\Delta v(s)\|,
\end{gathered}
$$

and we see that (46) for the derivative of (39) defines a bounded linear operator indeed [one may also check in the same way that the higher order derivatives 
of (39) are bounded multi linear operators]. Moreover, the norm of this operator is small (less than 1) for small $\sigma$, giving the required contraction.

The following result is a version of the well-known $\lambda$-lemma from the theory of finite-dimensional dynamical systems (see [15]; an infinite-dimensional version can be found in [16]). The advantage of our result is that we do not assume the spectral gap condition.

LEMMA 29 (Existence of the scaling limit). For some initial condition $u_{0}$ let the following limit relation hold:

$$
\lim _{t \uparrow \infty} e^{-\lambda t} T_{t} u_{0}=\sigma \phi
$$

Then there exists the limit

$$
\lim _{t \uparrow \infty} u\left(t ; e^{-\lambda t} u_{0}\right)=\psi(\sigma),
$$

where $u(t ; \xi)$ denotes the solution of (30) starting with the initial condition $u(0)=\xi$ and $\sigma \mapsto \psi(\sigma)$ is the equation of the invariant curve $\mathcal{Q}$ constructed in Lemma 25.

PROOF. By continuity of the nonlinear semigroup defined by (30), it is enough to prove that, for some small $\rho>0$,

$$
\lim _{t \uparrow \infty} u\left(t ; \rho e^{-\lambda t} u_{0}\right)=\psi(\sigma \rho),
$$

because if we denote $\theta=-\frac{1}{\lambda} \ln \rho>0$, then $u\left(t+\theta ; e^{-\lambda(t+\theta)} u_{0}\right)$ is the time $\theta$ shift of $u\left(t ; \rho e^{-\lambda t} u_{0}\right)$ and $\psi(\sigma)$ is the time $\theta$ shift of $\psi(\sigma \rho)$ [see (37)].

Write

$$
v(t)=e^{-\lambda t} u\left(t+\tau ; e^{-\lambda \tau} \rho u_{0}\right), \quad t \in[-\tau, 0] .
$$

By (30),

$$
v(t)=\rho e^{-\lambda(t+\tau)} T_{t+\tau} u_{0}+\int_{-\tau}^{t} e^{-\lambda t} T_{t-s} \circ f\left(e^{\lambda s} v(s)\right) d s .
$$

Let $v^{*}(t ; \rho \sigma)$ be the solution of (38), that is,

$$
v^{*}(t)=\rho \sigma \phi+\int_{-\infty}^{t} e^{-\lambda t} T_{t-s} \circ f\left(e^{\lambda s} v^{*}(s)\right) d s .
$$

We will prove that

$$
v(t)-v^{*}(t) \rightarrow 0
$$


as $\tau \rightarrow+\infty$, for any fixed $t \leq 0$. Then putting $t=0$ in (52) will give (49) and finish the proof of the lemma. In fact, we will prove that

$$
\sup _{t \in\left[-\tau^{\prime}, 0\right]}\|v(t)\| \rightarrow 0
$$

for an appropriately chosen $\tau^{\prime}$ which tends to $+\infty$ as $\tau \rightarrow+\infty$.

First, note that it follows from the existence of the finite limit (47) that $e^{-\lambda s} T_{s} u_{0}$ is uniformly bounded for all $s \geq 0$ :

$$
\sup _{s \geq 0}\left\|e^{-\lambda s} T_{s} u_{0}\right\| \leq L
$$

for some finite $L$. It is now easy to show that

$$
\|v(t)\| \leq 2 L \rho
$$

for all $\tau \geq 0$ and $t \in[-\tau, 0]$, provided $\rho$ is small enough. Indeed, this holds true at $t=-\tau$ for any $\tau$, and let $t_{0} \leq 0$ be the maximal value of $t$ for which (55) is still valid. If $t_{0}<0$, this means that $\left\|v\left(t_{0}\right)\right\|=2 L \rho$. Now, by (54), using estimates (35) and (33), we have, from (50),

$$
\left\|v\left(t_{0}\right)\right\| \leq L \rho+M K(2 L \rho)^{1+\delta} e^{\varepsilon t_{0}} \int_{-\tau}^{t_{0}} e^{(\lambda \delta-\varepsilon) s} d s \leq L \rho\left(1+\frac{2 M K}{\lambda \delta-\varepsilon}(2 L \rho)^{\delta}\right) .
$$

If $\rho$ was taken small enough, we get that $\left\|v\left(t_{0}\right)\right\|$ is strictly less than $2 L \rho$; hence $t_{0}=0$, which proves the claim.

Now take any $\tau^{\prime}<\tau$ such that $\tau^{\prime} \rightarrow+\infty$ as $\tau \rightarrow+\infty$. We have

$$
\begin{aligned}
& \left\|\int_{-\tau}^{-\tau^{\prime}} e^{-\lambda t} T_{t-s} \circ f\left(e^{\lambda s} v(s)\right) d s\right\| \\
& \quad \leq M K\left(\sup _{s \leq 0}\|v(s)\|\right)^{1+\delta} e^{\varepsilon t} \int_{-\tau}^{-\tau^{\prime}} e^{(\lambda \delta-\varepsilon) s} d s .
\end{aligned}
$$

By (36) and (55), this integral tends to zero as $\tau^{\prime} \rightarrow+\infty$, uniformly for any $t \leq 0$. The same conclusion can be made with respect to the integral

$$
\int_{-\tau}^{-\tau^{\prime}} e^{-\lambda t} T_{t-s} \circ f\left(e^{\lambda s} v^{*}(s)\right) d s:
$$

the estimate like (56) follows from (35) and (33), and the uniform boundedness of $v^{*}$ was proven in Lemma 1 [see (40); note that the upper bound on the norm on $v^{*}$ is also linear in $\rho$ in the present notation; i.e., $v^{*}$ also satisfies (55) with an appropriately chosen $L]$.

Hence, for any $t \in\left[-\tau^{\prime}, 0\right]$ we have the following from (50) and (51) [we use estimates (32), (55) and (35)]:

$$
\begin{aligned}
\left\|v(t)-v^{*}(t)\right\| \leq \xi\left(\tau^{\prime}\right)+ & M K(2 L \rho)^{\delta}\left(\int_{-\tau^{\prime}}^{0} e^{(\lambda \delta-\varepsilon) s} d s\right) \\
& \times \sup _{s \in\left[-\tau^{\prime}, 0\right]}\left\|v(s)-v^{*}(s)\right\|+o(1)_{\tau^{\prime} \rightarrow+\infty},
\end{aligned}
$$


where

$$
\xi\left(\tau^{\prime}\right)=\rho \sup _{s \in\left[\tau-\tau^{\prime}, \tau\right]}\left\|e^{-\lambda s} T_{s} u_{0}-\sigma \phi\right\| .
$$

Since $\xi\left(\tau^{\prime}\right) \rightarrow 0$ as $\tau-\tau^{\prime} \rightarrow+\infty$ [see (47)], it immediately follows from (57) that at sufficiently small $\rho$ the sought relation (53) holds, provided $\tau^{\prime}$ is chosen such that $\tau^{\prime} \rightarrow+\infty, \tau-\tau^{\prime} \rightarrow+\infty$.

Note that we never used in the proof of Lemma 29 (unlike in the proof of Lemma 25) the completeness of the space $\mathcal{X}$. Therefore, we may change Lemma 29 (in order to adapt it to the particular problem we consider in this paper) as follows.

LEMMA 30 (Scaling limit in a weaker norm). For any norm $\|\cdot\|_{1}$ which is weaker than the original norm $\|\cdot\|_{0}$ in $\mathcal{X}$, if the (linear) limit relation (47) holds in the norm $\|\cdot\|_{1}$ for some initial condition $u_{0}$, then the (nonlinear) limit relation (48) holds in the same norm, provided the following estimates are valid:

$$
\begin{gathered}
\|F(u)\|_{0} \leq K\|u\|_{0}^{\delta}, \\
\|F(u)\|_{1} \leq K\|u\|_{0}^{\delta}, \\
\left\|T_{t}\right\|_{0} \leq M e^{\lambda t}, \\
\left\|T_{t}\right\|_{1} \leq M e^{(\lambda+\varepsilon) t}
\end{gathered}
$$

with $\varepsilon<\lambda \delta$, where $F(u)$ is the derivative map from (32).

PROOF. The proof repeats the proof of Lemma 29 with the following modification: the estimate (55) (in the original $\|\cdot\|_{0}$-norm) now follows directly from (60). Then, it follows from (55), (59) and (61) that all the estimates of Lemma 29 remain unchanged in the norm $\|\cdot\|_{1}$. Finally, the required existence and uniform boundedness (in the original norm $\|\cdot\|_{0}$ and, hence, in the weaker norm $\|\cdot\|_{1}$ ) of the solution $v^{*}$ of the integral equation (38) are given by Lemma 25.

4. Some preliminary results for the proof of the main theorem. The proofs of Theorem 1 and Proposition 20 are based on two propositions (see Propositions 32 and 34 below) and on two lemmas stated and proved in Section 3 (Lemmas 25 and 30). We also use the following simple fact. 
LEMMA 31. For any $0<\gamma: \mathbb{R}^{d} \rightarrow \mathbb{R}$ continuous define the $\gamma$-norm by

$$
\|f\|_{\gamma}:=\|\gamma f\|,
$$

on $\{f$ continuous $: \gamma f$ is bounded $\}$. If $\gamma \in C_{0}$ and if $\mathcal{F}$ is a uniformly bounded family of functions, then the norm $\|\cdot\|_{\gamma}$ restricted to $\mathcal{F}$ is compatible with the topology of uniform convergence on compacts.

PROOF. First, assume that $f_{n}$ tends to zero uniformly on compacts as $n \uparrow \infty$. Since $\gamma \in C_{0}$ and by assumption $\left\|f_{n}\right\| \leq K, n \geq 1$, for some $K>0$, one can take a large ball $B \subset \mathbb{R}^{d}$ (depending on $\varepsilon$ ) such that

$$
\sup _{x \in \mathbb{R}^{d} \backslash B} \gamma(x) f_{n}(x)<\varepsilon, \quad n \geq 1 .
$$

Since $\gamma f_{n}$ also tends to zero uniformly on compacts as $n \uparrow \infty$, we can pick an $N=N(\varepsilon) \in \mathbb{N}$ such that

$$
\sup _{x \in B} \gamma(x) f_{n}(x)<\varepsilon, \quad n>N .
$$

Then, altogether we have

$$
\sup _{x \in \mathbb{R}^{d}} \gamma(x) f_{n}(x)<\varepsilon, \quad n>N,
$$

proving the $\gamma$-norm convergence for $f_{n}$.

Conversely, assume that $f_{n}$ tends to zero in $\gamma$-norm and fix an arbitrary nonempty ball $B \subset \mathbb{R}^{d}$. We have

$$
\sup _{x \in B} f_{n}(x) \leq C(\gamma, B) \sup _{x \in B} \gamma(x) f_{n}(x)
$$

with some $C(\gamma, B)>0$. The right-hand side of the last formula tends to zero as $n \uparrow \infty$ by assumption, thus the same is true for the left-hand side. This proves uniform convergence on compacts for $f_{n}$.

Let $\left\{S_{t}\right\}_{t>0}$ denote the semigroup corresponding to the operator $L+\beta-\lambda_{c}$ on $\mathbb{R}^{d}$ (and acting on $C_{b}$ ). Note that

$$
S_{t}=e^{-\lambda_{c} t} T_{t},
$$

where $\left\{T_{t}\right\}_{t>0}$ is the semigroup defined in (6).

Proposition 32 (Convergence for $S_{t}^{\phi} g$ in $\gamma$-norm). Assume that the condition in (ii-b) of Theorem $\mathrm{P}$ is satisfied, and furthermore let $0<\gamma \in C_{0}$. Then, for any $g \in C_{b}$,

$$
\lim _{t \uparrow \infty} S_{t}^{\phi} g=\langle g, \phi \tilde{\phi}\rangle \quad \text { in }\|\cdot\|_{\gamma}
$$


Proof. Since $L+\beta-\lambda_{c}$ is critical on $\mathbb{R}^{d}$, so is the $h$-transformed $(h=\phi)$ operator $\left(L+\beta-\lambda_{c}\right)^{\phi}$. Let $0<\chi$ and $\tilde{\chi}$ denote the eigenfunctions corresponding to the latter operator and to its adjoint, respectively. It is easy to see that $\chi=1$ and $\tilde{\chi}=\phi \tilde{\phi}$. In particular $\langle\chi, \tilde{\chi}\rangle=\langle\phi, \tilde{\phi}\rangle$. Note that the $\phi$-transformed operator

$$
\left(L+\beta-\lambda_{c}\right)^{\phi}=L+a \frac{\nabla \phi}{\phi} \cdot \nabla
$$

has no zeroth-order part (it is a diffusion generator). Using this along with the second part of [12], Theorem 4.4.9, we have that, for any $g \in C_{b}$ given,

$$
\lim _{t \uparrow \infty} S_{t}^{\phi} g=\langle g, \phi \tilde{\phi}\rangle,
$$

in the topology of uniform convergence on compacts. Our goal is to verify that this convergence holds also in $\|\cdot\|_{\gamma}$. Using Lemma 31 , it is enough to show that, for any $g \in C_{b}$ given,

$$
\mathcal{F}:=\left\{\left(S_{t}^{\phi}\right) g\right\}_{t \geq 0}
$$

is a uniformly bounded family of functions. Recalling that the $\phi$-transformed operator has no zeroth-order part and denoting the corresponding expectations by $\left\{\mathbb{E}_{x}^{\phi}\right\}_{x \in \mathbb{R}^{d}}$ we have

$$
\left(S_{t}^{\phi} g\right)(x)=\mathbb{E}_{x}^{\phi} g\left(Y_{t}\right),
$$

where $Y_{t}$ is the corresponding diffusion process. It then follows that

$$
\left\|S_{t}^{\phi}(g)\right\| \leq\|g\| .
$$

This completes the proof of the proposition.

We now choose a particular function $\gamma$ as follows. Let $h$ be a positive function satisfying the following:

1. $\left(L+\beta-\lambda_{c}\right)^{\phi} h \leq 0$ outside some compact set;

2. $h(x) \rightarrow \infty$ as $|x| \rightarrow \infty$.

The existence of such an $h$ follows by the recurrence of the diffusion corresponding to the operator $\left(L+\beta-\lambda_{c}\right)^{\phi}$ and from the following theorem which we feel is of independent interest. (For the proof see Appendix A.)

THEOREM 3 (Necessary condition for recurrence). Let $L$ be as in (1), and assume that it corresponds to a recurrent diffusion process $Y$. Given any positive $R_{1}$ and any function $p(x)$ which tends to infinity as $|x| \rightarrow+\infty$, there exists a supersolution on $|x| \geq R_{1}$, that is, a positive $C^{2, \eta}$-function $U(x)$ such that

$$
L U \leq 0 \quad \text { on }|x| \geq R_{1},
$$


converging to infinity as $|x| \rightarrow+\infty$, asymptotically slower than $p$ :

$$
\lim _{r \rightarrow+\infty} \inf _{|x|=r} U(x)=\infty, \quad \lim _{r \rightarrow+\infty} \sup _{|x|=r} \frac{U(x)}{p(x)}=0 .
$$

The existence of such a supersolution growing to infinity is known as a sufficient condition for the recurrence of $L$ (see [12], Theorem 6.1.2). Our result here shows that this is also a necessary condition for recurrence [earlier it was known only in the one-dimensional case - then the statement follows easily from [12], Theorem 5.1.1(i)].

REMARK 33. By the previous theorem, $h$ can be chosen to be arbitrarily slowly growing. This fact is used in the proof of Theorem 1 .

Using the above $h$, we define $\gamma$ as follows. Let

$$
\gamma:=1 / \hat{h}, \quad \text { where } \hat{h}=h+K
$$

and $K$ is a positive constant to be fixed later. Then, obviously, $0<\gamma \in C_{0}$.

Proposition 34 (Estimate for $S_{t}^{\phi}$ in $\gamma$-norm). Assume that $L+\beta-\lambda_{c}$ is critical with the ground state $\phi$ and let $\left\{S_{t}\right\}_{t>0}$ be as in Proposition 32. For any $\varepsilon>0$,

$$
\left\|S_{t}^{\phi}\right\|_{\gamma} \leq e^{\varepsilon t}, \quad t>0,
$$

if $K=K_{\varepsilon}$ is large enough [ $K$ is defined in (63)].

Proof. By a simple computation, the statement is equivalent to

$$
\left\|S_{t}^{\phi \hat{h}}\right\| \leq e^{\varepsilon t}, \quad t>0 .
$$

Recall that $\left(L+\beta-\lambda_{c}\right)^{\phi}$ has no zeroth-order part. Since the zeroth-order term of $\left(L+\beta-\lambda_{c}\right)^{\phi \hat{h}}$ is

$$
\frac{1}{\hat{h}}\left(L+\beta-\lambda_{c}\right)^{\phi} \hat{h}=\frac{1}{\hat{h}}\left(L+\beta-\lambda_{c}\right)^{\phi} h=: V,
$$

we have that

$$
V \leq 0
$$

outside a compact set by the first assumption on $h$. Also, if $K$ is large enough, we can obviously guarantee that

$$
V \leq \varepsilon \quad \text { on } \mathbb{R}^{d} .
$$

The estimate under (65) now follows from this and (6) with $g=1$ and $\beta$ replaced by $V$ (but now with $\mathbb{E}$ corresponding to $L_{0}^{\phi \hat{h}}$ ). 


\section{Proof of Theorems 1 and 2 and Proposition 20.}

Proof OF THEOREM 1. The strategy of the proof is as follows. We will show that the scaling limit exists in law for $X^{\phi}$. More precisely, we will prove that, for $\mu \in \mathcal{M}^{1 / \gamma}$ with $\gamma$ given by (63),

$$
\lim _{t \uparrow \infty} E_{\mu}^{\phi} \exp \left\langle e^{-\lambda_{c} t} X_{t}^{\phi},-g\right\rangle=E \exp \left\langle Z_{\mu},-g\right\rangle, \quad g \in C_{b}^{+},
$$

with some random measure $Z_{\mu}$ having the form $Z_{\mu}=N_{\mu}^{\phi} \phi \tilde{\phi} d x$, where the random variable $N_{\mu}^{\phi}$ is determined by (12) [or by (28) for a general nonlinearity] and enjoys the properties stated in the theorem [note that in (12) or (28) the curve $\sigma \mapsto \psi(\sigma)$ is now replaced by a new curve corresponding to the $\phi$-transformed dynamics, i.e., to $T_{t}^{\phi}$ and $\alpha^{\phi}$ or $f^{\phi}$ ]. Having shown this, it will follow from the definition of the $h$-transform that (11) holds for $X$ starting with the measure $v:=\frac{1}{\phi} \mu$ [a simple computation shows that (12) holds for the original curve $\sigma \mapsto \psi(\sigma)$ when going back to $X$ ]. That is, when $\phi(h+K) v$ [where $h, K$ are from (63)] is a finite measure. Putting this together with the fact that $h$ can be chosen to be arbitrarily slowly growing by Theorem 3, we will have that (11) holds true whenever $v \in \mathcal{M}^{\phi}$. It will also follow that (66) is satisfied for $X$ in place of $X^{\phi}$ and $C_{b}^{+}$replaced by the class of all continuous $g$ 's with $g \leq$ const $\cdot \phi \hat{h}=$ const $\cdot \phi(h+K)$. In particular, (66) will hold for $X^{\phi}$ replaced by $X$, provided that $\phi$ is bounded away from zero [recall that $h(x) \rightarrow \infty$ as $|x| \rightarrow \infty$ ]. This will prove (14).

Now we show (66). To do this, let us summarize what we already know about the nonlinear semigroup corresponding to $X^{\phi}$. First, concerning the linear part of the semigroup, $T_{t}^{\phi}$, we know that the rescaled semigroup $S_{t}^{\phi}$ corresponding to $\left(L+\beta-\lambda_{c}\right)^{\phi}$ has the following properties:

(a) $\left(L+\beta-\lambda_{c}\right)^{\phi}$ is a diffusion generator, that is, $\left(\beta-\lambda_{c}\right)^{\phi}=0$, and the ground state $\phi$ transforms into 1 .

(b) By Proposition 32, for any $g \in C_{b}, S_{t}^{\phi} g$ has the limit $\langle g, \phi \tilde{\phi}\rangle$ in $\|\cdot\|_{\gamma}$.

(c) By Proposition 34, $S_{t}^{\phi}$ satisfies the exponential estimate under (64). Also, $\left\|S_{t}^{\phi}\right\| \leq 1$ since $\left\{S_{t}^{\phi}\right\}_{t \geq 0}$ is a diffusion semigroup (see the end of the proof of Proposition 32).

In addition to the linear part of the semigroup, we have to control the nonlinear term

$$
f^{\phi}(u)=\alpha^{\phi} u^{2}
$$

Here $\alpha^{\phi}=\alpha \phi$. Thus, for the derivative map

$$
F(u): d u \mapsto 2 \alpha \phi u d u,
$$


we have (recall that $\|\cdot\|$ denotes the supremum norm)

$$
\|2 \alpha \phi u d u\|_{\gamma} \leq\|2 \alpha \phi u\|\|d u\|_{\gamma}
$$

That is,

$$
\|F(u)\|_{\gamma} \leq\|2 \alpha \phi u\| \leq 2\|\alpha \phi\|\|u\| .
$$

By the same computation, also

$$
\|F(u)\| \leq 2\|\alpha \phi\|\|u\| .
$$

Altogether, working with the nonlinear dynamics corresponding to $X^{\phi}$ and with $\|\cdot\|_{\gamma}$, we are in the position to implement the invariant curve method of Section 3. More precisely, we are going to apply Lemma 30 with $\mathcal{X}=C_{b}$, $\mathcal{X}^{+}=C_{b}^{+},\|\cdot\|_{0}=\|\cdot\|$ and $\|\cdot\|_{1}=\|\cdot\|_{\gamma}$; where furthermore $T_{t}$ and $\phi$ are replaced by $T_{t}^{\phi}$ and the function 1 . [Clearly, $1 \in \operatorname{int}\left(C_{b}^{+}\right)$in sup-norm topology.] Let $0 \leq u(t, g, \cdot)$ denote the solution of (5) or (25) [but $L, \beta$ and $f$ replaced with $\left(L+\beta-\lambda_{c}\right)^{\phi},\left(\beta-\lambda_{c}\right)^{\phi}=0$ and $f^{\phi}$, respectively] with $u(0, \cdot)=g(\cdot)$. Let furthermore $\sigma \mapsto \psi(\sigma)$ be the invariant curve constructed in Section 3. Working with $\|\cdot\|_{\gamma}$ and using the discussion at the beginning of this paragraph along with Lemma 30 of Section 3, (3) and (5) or (25) applied to the $\phi$-transformed setting yields

$$
\begin{aligned}
E_{\mu}^{\phi} \exp \left\langle e^{-\lambda_{c} t} X_{t}^{\phi},-g\right\rangle & =\exp \left\langle\mu,-u\left(t, e^{-\lambda_{c} t} g\right)\right\rangle \\
& =\exp \left\langle\mu / \gamma,-\gamma u\left(t, e^{-\lambda_{c} t} g\right)\right\rangle \\
& \rightarrow \exp \langle\mu / \gamma,-\gamma \psi(\langle g, \phi \tilde{\phi}\rangle)\rangle \quad \text { as } t \rightarrow \infty,
\end{aligned}
$$

provided $\mu \in \mathcal{M}^{1 / \gamma}, \gamma g \in C_{b}^{+}$(and in particular for $g \in C_{b}^{+}$). That is,

$$
E_{\mu}^{\phi} \exp \left\langle e^{-\lambda_{c} t} X_{t}^{\phi},-g\right\rangle \longrightarrow \exp \langle\mu,-\psi(\langle g, \phi \tilde{\phi}\rangle)\rangle \quad \text { as } t \rightarrow \infty .
$$

Now let us fix a $\mu \in \mathcal{M}^{1 / \gamma}$. Note that the functional

$$
\Psi_{\mu}(g):=\exp \langle\mu,-\psi(\langle g, \phi \tilde{\phi}\rangle)\rangle
$$

defined on $C_{b}^{+}$is positive definite (for the definition of positive definiteness see, e.g., the proof of Theorem A in [8]), because it is the pointwise limit of functionals possessing this property. Moreover, $\Psi_{\mu}$ is continuous with respect to bounded pointwise convergence, since $\phi \tilde{\phi} d x \in \mathcal{M}$ by assumption. Also, $\Psi_{\mu}(0)=1$, because $\psi(0)=0$. It follows from these properties by a standard result (see the proof of Theorem A1 in [8]; see also [4], Lemma 3.1), that $\Psi_{\mu}$ is the Laplace functional of a random measure; that is, there exists a random measure $Z_{\mu}$ such that

$$
E e^{\left\langle Z_{\mu},-g\right\rangle}=\exp \langle\mu,-\psi(\langle g, \phi \tilde{\phi}\rangle)\rangle
$$


for $g \in C_{b}^{+}$. Therefore, altogether,

$$
E_{\mu} \exp \left\langle e^{-\lambda_{c} t} X_{t}^{\phi},-g\right\rangle \longrightarrow E e^{\left\langle Z_{\mu},-g\right\rangle} \quad \text { as } t \rightarrow \infty,
$$

whenever $g \in C_{b}^{+}$. That is, $e^{-\lambda_{c} t} X_{t}^{\phi}$ converges to $Z_{\mu}$ in law.

To identify $Z_{\mu}$, note that if $N_{\mu}^{\phi}$ is a nonnegative random variable satisfying (12) (again, the existence of such a random variable is guaranteed by the positive definiteness and continuity of $s \mapsto \exp \langle\mu, \psi(s)\rangle)$, then the random measure

$$
Z_{\mu}^{*}:=N_{\mu}^{\phi} \phi \tilde{\phi}(x) d x
$$

clearly satisfies (67) and thus by uniqueness $Z_{\mu}=Z_{\mu}^{*}$.

Using the fact that $\psi^{\prime}(0)=\phi$, (13) follows. [To do this rigorously, recall that $\psi^{\prime}(0)=\phi$ means that $\lim _{s \rightarrow 0} \psi(s) / s=\phi$ in $\|\cdot\|_{\gamma}$. Since $\mu \in \mathcal{M}^{1 / \gamma}$, we can use uniform convergence to conclude (13).]

Finally, we show that $N_{\mu}^{\phi}$ is nondegenerate. Suppose to the contrary that $N_{\mu}^{\phi}=$ $\mathbf{E} N_{\mu}^{\phi}=\langle\mu, \phi\rangle$ with $P_{\mu}$-probability 1 . By (12) this would imply that $\psi(s)=s \phi$ for $s>0$. But this is impossible because $\psi$ is invariant with respect to the nonlinear system (75). Consequently $N_{\mu}^{\phi}$ is indeed nondegenerate. This completes the proof of Theorem 1.

PROOF OF PROPOSITION 20. The proof is the same as the proof of Theorem 1 except the following. For the general nonlinearity (21) we have

$$
f^{\phi}(u)=\alpha^{\phi} u^{2}+\int_{0}^{\infty}\left(e^{-k u}-1+k u\right) n^{\phi}(x, d k),
$$

where $n^{\phi}(x, d k)=\phi(x)^{-1} n\left(x, \phi(x)^{-1} d k\right)$. The derivative map is

$$
F(u): d u \mapsto\left[2 \alpha \phi u+\int_{0}^{\infty} k\left(1-e^{-k u}\right) n^{\phi}(x, d k)\right] d u .
$$

Here, we have

$$
\begin{aligned}
& \left\|\int_{0}^{\infty} k\left(1-e^{-k u}\right) n^{\phi}(x, d k) d u\right\|_{\gamma} \\
& \quad \leq \sup _{x \in \mathbb{R}^{d}} \int_{0}^{\infty}\left[u(x) \phi(x) k^{2} \wedge u^{\delta}(x) \phi^{\delta}(x) k^{1+\delta}\right] n(x, d k)\|d u\|_{\gamma} .
\end{aligned}
$$

By (26),

$$
\|F(u)\|_{\gamma}=O\left(\|u\|+\|u\|^{\delta}\right)
$$

and, analogously,

$$
\|F(u)\|=O\left(\|u\|+\|u\|^{\delta}\right) .
$$


These estimates are enough to obtain the results of Section 3, so the rest of the proof for general nonlinearity goes exactly the same way as in the case $f(u)=\alpha u^{2}$.

Proof of ThEOREM 2. The proof of Theorem 2 is very similar to that of Theorem 1. We use the results of Section 3 in exactly the same way as in the case of Theorem 1, but we have to replace the "linear result" with an analogous result for the singular setting and moreover to replace the pde setting of Propositions 32 and 34 by using the integral equation (8). Fix a bounded continuous $g$, and set

$$
u(x, t):=E_{\delta_{x}}^{\sin }\left\langle X_{t}, g\right\rangle, \quad x \in \mathbb{R}, t \geq 0 .
$$

Using (8), it is standard to verify the following integral equation for the expectations [see [7], formula (91)]:

$$
u(x, t)=\int_{\mathbb{R}} d y p(t, y-x) g(y)+\int_{0}^{t} d s p(t-s, x) u(0, s),
$$

$x \in \mathbb{R}, t \geq 0$. [Symbolically, $u_{t}=\frac{1}{2} \Delta u+\delta_{0} u$ with $u(x, 0)=g$.] Analogously to the section preceding (7), let us define now the semigroup $\left\{T_{t}\right\}_{t \geq 0}$ by

$$
\left(T_{t} g\right)(\cdot):=u(\cdot, t) .
$$

(The semigroup property can be checked by direct calculation.) By Theorem EF then, we know that $e^{-t / 2} T_{t} g$ has a pointwise limit as $t \rightarrow \infty$ for any bounded continuous $g: \mathbb{R} \mapsto \mathbb{R}_{+}$.

Let $\phi(x):=e^{-|x|}$ (recall that the function $x \mapsto e^{-|x|}$ plays the role of the ground state; this justifies our notation.) Define the $\phi$-transformed semigroup by

$$
T_{t}^{\phi}(g):=e^{|x|} T_{t}\left(e^{-|x|} g\right) \quad \text { for } e^{-|x|} g \in C_{b}^{+} .
$$

Define also $S_{t}^{\phi}(g):=e^{-t / 2} T_{t}^{\phi}(g)$. Let $\mu=\delta_{x}$ and rewrite (9):

$$
\lim _{t \uparrow \infty}\left(S_{t} g\right)(x)=e^{-|x|}\left\langle e^{-|x|}, g\right\rangle, \quad g \in C_{b}^{+} .
$$

Let $G:=e^{|x|} g$. Then

$$
\lim _{t \uparrow \infty}\left(S_{t}^{\phi} G\right)(x)=\left\langle e^{-2|x|}, G\right\rangle .
$$

Now (70) holds for every $G$ satisfying $e^{-|x|} G \in C_{b}^{+}$. In particular, (70) holds for every $G \in C_{b}^{+}$. We now show that this convergence is uniform on compacts. Let us fix a $K \subset \mathbb{R}$ compact. We must show that, for $g \in C_{b}^{+}$,

$$
e^{-t / 2} e^{|x|} u(x, t) \rightarrow C(g) \quad \text { as } t \uparrow \infty
$$

uniformly for $x \in K$, where $C(g):=\left\langle e^{-|x|}, g\right\rangle$. Exploiting the notation $u_{x}(t):=$ $u(x, t)$ and $p_{x}(t):=p(t, x)$, the Laplace transform of (69) (with respect to $t$ ) is

$$
\widehat{u_{x}}(\lambda)=\int_{\mathbb{R}} d y \widehat{p_{y-x}}(\lambda) g(y)+\widehat{p_{x}}(\lambda) \widehat{u_{0}}(\lambda),
$$


where $\widehat{u_{x}}$ and $\widehat{p_{x}}$ denote the Laplace transforms of $u_{x}$ and $p_{x}$, respectively. Using (72), the Laplace transform of the left-hand side of (71) is

$$
\begin{aligned}
e^{|x|} \widehat{u_{x}}\left(\lambda+\frac{1}{2}\right) & =e^{|x|} \int_{\mathbb{R}} d y \widehat{p_{y-x}}\left(\lambda+\frac{1}{2}\right) g(y)+e^{|x|} \widehat{p_{x}}\left(\lambda+\frac{1}{2}\right) \widehat{u_{0}}\left(\lambda+\frac{1}{2}\right) \\
& =: M(x, \lambda)+N(x, \lambda) \widehat{u_{0}}\left(\lambda+\frac{1}{2}\right) .
\end{aligned}
$$

By continuity, $M$ is bounded on $K \times[0, \varepsilon]$. Let

$$
a:=\inf _{\substack{x \in K \\ \lambda<\varepsilon}} M(x, \lambda) \quad \text { and } \quad A:=\sup _{\substack{x \in K \\ \lambda<\varepsilon}} M(x, \lambda) .
$$

In the proof of Theorem 4(b) in [7] we have shown that

$$
\widehat{u_{0}}\left(\lambda+\frac{1}{2}\right) \sim C(g) \frac{1}{\lambda} \quad \text { as } \lambda \downarrow 0
$$

and that

$$
N(x, \lambda) \rightarrow 1 \quad \text { as } \lambda \downarrow 0,
$$

for each $x \in \mathbb{R}$. We now show that in fact the convergence in (74) is uniform on $K$. To see this, note that $N(x, \lambda)$ is continuous in $x$ by monotone convergence. The uniformity of the limit in (74) thus follows by Dini's theorem. Let

$$
b(\lambda):=\inf _{x \in K} N(x, \lambda) \quad \text { and } \quad B(\lambda):=\sup _{x \in K} N(x, \lambda) .
$$

Then we have

$$
a+b(\lambda) \widehat{u_{0}}\left(\lambda+\frac{1}{2}\right) \leq e^{|x|} \widehat{u_{x}}\left(\lambda+\frac{1}{2}\right) \leq A+B(\lambda) \widehat{u_{0}}\left(\lambda+\frac{1}{2}\right),
$$

with

$$
\lim _{\lambda \downarrow 0} b(\lambda)=\lim _{\lambda \downarrow 0} B(\lambda)=1 .
$$

Using this, (73) and a well-known Tauberian theorem [10], formula (13.5.22) along with the monotonicity of the Laplace transform, it follows that (71) holds uniformly on $K$.

Similarly to the proof of Theorem 1, in order to conclude convergence in $\gamma$-norm, we have to show that $\left\{S_{t}^{\phi} G, t \geq 0\right\}$ is a uniformly bounded family, for every given $G \in C_{b}^{+}$. Let $G \in C_{b}^{+}$with $\|\bar{G}\|=K$. Since $\left\langle e^{-2|x|}, 1\right\rangle=1$, we have

$$
\lim _{t \uparrow \infty}\left(S_{t}^{\phi} G\right)(x) \leq K .
$$

Consequently,

$$
\left\|S_{t}^{\phi} G\right\| \leq K^{*} \quad \text { for all } t \geq 0
$$


with some $K^{*}>K$; that is, $\left\{S_{t}^{\phi} G, t \geq 0\right\}$ is a uniformly bounded family, for every given $G \in C_{b}^{+}$. Thus, we have shown convergence in $\gamma$-norm for any $\gamma \in C_{0}$.

Now choose

$$
\gamma:=\phi=e^{-|x|} .
$$

We look for a substitute of Proposition 34 for the nonregular setting. By Theorem 4(a) in [8] we have that

$$
\lim _{t \uparrow \infty} e^{-t / 2}\left\|T_{t}\right\|=2 .
$$

A simple calculation reveals that

$$
\left\|T_{t}^{\phi}\right\|_{\phi}=\left\|T_{t}\right\|
$$

Therefore, also

$$
\lim _{t \uparrow \infty} e^{-t / 2}\left\|T_{t}^{\phi}\right\|_{\phi}=2
$$

and consequently

$$
e^{-t / 2}\left\|T_{t}^{\phi}\right\|_{\phi} \leq K \quad \text { for all } t \geq 0,
$$

with some $K>2$. This gives the required estimate for the $\phi$-transformed linear semigroup.

Finally, the $\phi$-transformed superdiffusion $X^{\phi}$ can be defined in the usual way: it will correspond to the integral equation

$$
u(\cdot, t)=T_{t}^{\phi} g-\int_{0}^{t} d s T_{t-s}^{\phi}\left(\alpha \phi u^{2}(\cdot, s)\right) .
$$

The rest of the proof is virtually identical with the last part of the proof of Theorem 1 (by setting $\lambda_{c}=1 / 2$ and $\phi=e^{-|x|}$ in that proof), except that the convergence of the $\phi$-transformed Laplace functional now holds for all $g$ 's with $\phi g \in C_{b}^{+}$(recall that $\gamma=\phi$ ), thus yielding convergence far all nonnegative bounded continuous functions when going back to the original Laplace functional.

\section{APPENDIX A}

ProOF OF THEOREM 3. Let $Y$ denote the diffusion corresponding to $L$ on $\mathbb{R}^{d}$ with probabilities $\left\{\mathbb{P}_{x}, x \in \mathbb{R}^{d}\right\}$. Let $\tau_{R}:=\inf \left\{t \geq 0|| Y_{t} \mid=R\right\}$. Using Itô's formula, it is immediate that, for any fixed $R_{0}>0, \bar{U}\left(x, R_{0}, R\right):=\mathbb{P}_{x}\left(\tau_{R_{0}}>\tau_{R}\right)$ is the unique solution to the boundary value problem

$$
\begin{aligned}
L u & =0 \quad \text { at } R_{0} \leq|x| \leq R, \\
u=0 \quad \text { at }|x| & =R_{0} \quad \text { and } \quad u=1 \quad \text { at }|x|=R .
\end{aligned}
$$


By the recurrence of $Y, \bar{U}\left(x, R_{0}, R\right)$ tends to zero in the layer $|x| \in\left[R_{0}, R_{0}+C\right]$, as $R \rightarrow+\infty$, for any fixed finite $C>0$.

Note that

$$
0<\bar{U}<1 \text { for }|x| \in\left(R_{0}, R\right) .
$$

Let $(r, \varphi)$ denote spherical coordinates (i.e., $r=|x|)$. By the Hopf maximum principle (see [12], Theorem 3.2.5),

$$
\bar{U}_{r}^{\prime}\left(x ; R_{0}, R\right)>0 \quad \text { both at } r=R_{0} \text { and at } r=R .
$$

Next, we show that

$$
\left.\bar{U}_{r}^{\prime}\right|_{r=R_{0}} \leq\left. K\left(R_{0}\right) \sup _{\varphi} \bar{U}\right|_{r=R_{0}+1},
$$

where the constant $K$ depends (continuously) only on the coefficients of $L$ at $r \in\left[R_{0}, R_{0}+1\right]$; that is, it is independent of the position of the outer boundary $(r=R)$. Hence,

$$
\left.\bar{U}_{r}^{\prime}\right|_{r=R_{0}} \rightarrow 0 \quad \text { as } R \rightarrow+\infty .
$$

To prove inequality (78), just note that

$$
U^{*}(x)=U^{*}(r)=\frac{1-e^{-K\left(r-R_{0}\right)}}{1-e^{-K}}
$$

is a supersolution for a sufficiently large $K$ :

$$
L U^{*}=-K^{2} e^{-K\left(r-R_{0}\right)}(\nabla r, a \nabla r)+O(K)<0,
$$

and, by construction, $U^{*}\left(r=R_{0}\right)=0, U^{*}\left(r=R_{0}+1\right)=1$. Hence, the product $U^{*}(x)\left(\left.\sup _{\varphi} \bar{U}\right|_{r=R_{0}+1}\right)$ is a supersolution with the boundary values at $r=R_{0}$ and $r=R_{0}+1$ not smaller than those of $\bar{U}$. By the elliptic comparison principle, this implies that

$$
U^{*}(x)\left(\left.\sup _{\varphi} \bar{U}\right|_{r=R_{0}+1}\right) \geq \bar{U}(x) \quad \text { at } r \in\left[R_{0}, R_{0}+1\right]
$$

and, in particular, $\bar{U}_{r}^{\prime}\left(r=R_{0}\right) \leq U_{r}^{* \prime}\left(r=R_{0}\right)\left(\left.\sup _{\varphi} \bar{U}\right|_{r=R_{0}+1}\right)$, which proves (78). When using this inequality we will always assume that $K\left(R_{0}\right)$ grows monotonically with $R_{0}$.

To prove our theorem on the existence of supersolutions, we use an inductive construction: we produce a sequence increasing to infinity $R_{1}<R_{2}<\cdots$ and, having built a supersolution $U^{(q)}$ defined at $R_{1} \leq r \leq R_{q}$, we continue it to the domain $r \leq R_{q+1}$, where $R_{q+1}>R_{q}$ may be taken arbitrarily large (though finite). The new supersolution $U^{(q+1)}$ will coincide with $U^{(q)}$ at $r \leq R_{q}-\delta_{q}$, where $\delta_{q}$ can be taken arbitrarily small. So this procedure, indeed, gives in the limit a supersolution defined at all $r \geq R_{1}$ [recall that $(r, \varphi)$ denote spherical coordinates, $r=|x|]$. 
At the first step $(q=2)$ we take

$$
U^{(2)}(x)=\bar{U}\left(x ; R_{1}, R_{2}\right) ;
$$

that is, it is the solution of the boundary-value problem (75) for an arbitrary $R_{2}>R_{1}$.

Let us now assume that we have the supersolution $U^{(q)}$ defined at $R_{1} \leq r \leq R_{q}$ such that

$$
U^{(q)}\left(R_{q}, \varphi\right) \equiv u_{q}=\mathrm{const}
$$

and

$$
\inf _{\varphi} U_{r}^{(q)^{\prime}}\left(R_{q}, \varphi\right)>0 .
$$

By construction [see (77)], these two requirements are satisfied at $q=2$, with $u_{2}=1$.

Denote

$$
\alpha(\varphi) \equiv U_{r}^{(q) \prime}\left(R_{q}, \varphi\right) .
$$

Take any $R_{q+1}>R_{q}+1$ such that

$$
K\left(R_{q}\right) \sup _{|x| \in\left[R_{q}-1, R_{q}+1\right]} \bar{U}\left(x ; R_{q}-1, R_{q+1}\right)<\frac{1}{\sup _{\varphi} \alpha(\varphi)} \inf _{\varphi} \alpha^{2}(\varphi) .
$$

Choose a sufficiently small $\delta_{q}>0$ (arbitrarily small, in fact) and take the solution $\bar{U}\left(x ; R_{q}-\delta_{q}, R_{q+1}\right)$ of the boundary-value problem (75). For brevity, we denote $\bar{U}(x) \equiv \bar{U}\left(x ; R_{q}-\delta_{q}, R_{q+1}\right)$ below. We also use the notation

$$
\beta(\varphi) \equiv \bar{U}_{r}^{\prime}\left(R_{q}-\delta_{q}, \varphi\right) .
$$

Since $\bar{U}\left(x ; R_{q}-\delta_{q}, R_{q+1}\right) \leq \bar{U}\left(x ; R_{q}-1, R_{q+1}\right)$ for any $\delta_{q} \in[0,1]$, it follows [see (77), (78) and (81)] that

$$
0<\inf _{\delta_{q} \in[0,1]} \inf _{\varphi} \beta(\varphi) \leq \sup _{\delta_{q} \in[0,1]} \sup _{\varphi} \beta(\varphi)<\frac{1}{\sup _{\varphi} \alpha(\varphi)} \inf _{\varphi} \alpha^{2}(\varphi) .
$$

This inequality allows us to find such constants $\lambda$ and $A$ that

$$
\lambda>\sup _{\delta_{q} \in[0,1]} \sup _{\varphi} \frac{\beta(\varphi)}{\alpha(\varphi)}
$$

and

$$
\inf _{\varphi} \alpha(\varphi)>A>\lambda \sup _{\varphi} \alpha(\varphi)
$$


Now let us define

$$
U^{(q+1)}(x)= \begin{cases}U^{(q)}(x), & \text { for }|x| \leq R_{q}-\delta_{q}, \\ \bar{U}(x)+u_{q}-A \delta_{q}, & \text { for } R_{q+1} \geq|x| \geq R_{q}, \\ u_{q}+\left(U^{(q)}(x)-u_{q}\right)\left(1-\xi_{1}\right) & \\ \quad+\left(\bar{U}(x)-A \delta_{q}\right) \xi_{2}, & \text { for }|x| \in\left[R_{q}-\delta_{q}, R_{q}\right],\end{cases}
$$

where $A$ is the constant from (84) and $\xi_{1,2}$ are some $C^{2, \eta}$-functions of $z \equiv$ $\left(r-R_{q}+\delta_{q}\right) / \delta_{q}$ such that

$$
\xi(z) \equiv \begin{cases}0, & \text { at } z \leq 0 \\ 1, & \text { at } z \geq 1\end{cases}
$$

and

$$
0<\xi(z)<1 \quad \text { at } z \in(0,1) .
$$

Moreover,

$$
\xi^{\prime}(z)>0 \quad \text { at } z \in(0,1) .
$$

In the rest of this section, any $C^{2, \eta}$-function satisfying (86)-(88) will be called nice.

Obviously, the function $U^{(q+1)}$ defined by (85) is $C^{2, \eta}$ and it is a supersolution [i.e., it satisfies (62)] for $r \leq R_{q}-\delta_{q}$ and $r \geq R_{q}$. So, we must check that it is a supersolution in the layer $R_{q}-\delta_{q} \leq r \leq R_{q}$ too, for an appropriate choice of the "gluing" functions $\xi_{1,2}$. In this layer, the inequality to check is

$$
\begin{aligned}
& -\left(U^{(q)}(x)-u_{q}\right) L \xi_{1}-2\left(\nabla U^{(q)}, a \nabla \xi_{1}\right) \\
& \quad+\left(\bar{U}(x)-A \delta_{q}\right) L \xi_{2}+2\left(\nabla \bar{U}(x), a \nabla \xi_{2}\right) \leq 0 .
\end{aligned}
$$

Note that at $|x| \in\left[R_{q}-\delta_{q}, R_{q}\right]$ we have

$$
\begin{aligned}
\left.\nabla U^{(q)}\right|_{x=(r, \varphi)} & =\alpha(\varphi) \nabla r+O\left(\delta_{q}\right), \\
\left.\nabla \bar{U}\right|_{x=(r, \varphi)} & =\beta(\varphi) \nabla r+O\left(\delta_{q}\right), \\
U^{(q)}(r, \varphi) & =u_{q}-\left[\alpha(\varphi)+O\left(\delta_{q}\right)\right]\left(R_{q}-r\right), \\
\bar{U}(r, \varphi) & =\left[\beta(\varphi)+O\left(\delta_{q}\right)\right]\left(r-R_{q}+\delta_{q}\right) .
\end{aligned}
$$

Also, it is easy to see that

$$
\nabla \xi=\frac{1}{\delta_{q}} \xi^{\prime} \nabla r
$$

and

$$
L \xi=\frac{1}{\delta_{q}^{2}} \xi^{\prime \prime}(\nabla r, a \nabla r)+O\left(\frac{1}{\delta_{q}}\right) \xi^{\prime} .
$$


Plugging this into (89) we arrive at the following condition which must be fulfilled at all $\varphi$ and at all $z \in[0,1]$ :

$$
\begin{aligned}
& {\left[(1-z) \xi_{1}^{\prime \prime}-2\left(1+O\left(\delta_{q}\right)\right) \xi_{1}^{\prime}\right](z)} \\
& \quad \leq\left[\frac{A-\beta(\varphi) z+O\left(\delta_{q}\right)}{\alpha(\varphi)+O\left(\delta_{q}\right)} \xi_{2}^{\prime \prime}-2 \frac{\beta(\varphi)+O\left(\delta_{q}\right)}{\alpha(\varphi)+O\left(\delta_{q}\right)} \xi_{2}^{\prime}\right](z) .
\end{aligned}
$$

Since $\xi_{1,2}^{\prime}$ is nonnegative by assumption, and since $\delta_{q}$ may be taken as small as necessary, it is sufficient that, for some sufficiently small $v$,

$$
\begin{aligned}
& (1-z) \xi_{1}^{\prime \prime}(z)-(2-v) \xi_{1}^{\prime}(z) \\
& \quad \leq\left(1-v \operatorname{sign}\left(\xi_{2}^{\prime \prime}(z)\right)\right) \frac{A-\beta(\varphi) z}{\alpha(\varphi)} \xi_{2}^{\prime \prime}(z)-(2-v) \lambda \xi_{2}^{\prime}(z),
\end{aligned}
$$

where $\lambda$ is the constant from (83), (84) [recall that $A>\beta(\varphi)$ by (83), (84)]. Write

$$
\xi_{0}(z)=\frac{\xi_{1}(z)-\lambda \xi_{2}(z)}{1-\lambda}
$$

By (83), (84), if $v$ is sufficiently small, then to satisfy the inequality (91) it is enough to require that

$$
(1-z) \xi_{0}^{\prime \prime}(z)-(2-v) \xi_{0}^{\prime}(z) \leq \chi(z) \xi_{2}^{\prime \prime}(z),
$$

where

$$
\chi(z)= \begin{cases}\chi_{+}, & \text {for } \xi_{2}^{\prime \prime}(z)>0 \\ \chi_{-}, & \text {for } \xi_{2}^{\prime \prime}(z)<0\end{cases}
$$

for some appropriately chosen constants $\chi_{ \pm}$which may be taken such that

$$
0<\chi_{+}<\chi_{-}<1 \text {. }
$$

Let us now take a smooth function $\psi(z)$ with zeros at 0 , at 1 and at some $\zeta \in(0,1)$. Let $\psi(z)>0$ at $0<z<\zeta$ and $\psi(z)<0$ at $\zeta<z<1$. Also, let

$$
\int_{0}^{\zeta} \psi(z) d z=-\int_{\zeta}^{1} \psi(z) d z=1
$$

Write

$$
I_{v}^{+}=\int_{0}^{\zeta} \psi(z)(1-z)^{(1-v)} d z, \quad I_{v}^{+}=-\int_{\zeta}^{1} \psi(z)(1-z)^{(1-v)} d z .
$$

Let

$$
\xi_{2}(z)=\frac{1}{I_{0}^{+}+I_{0}^{-}} \int_{0}^{z}(z-s) \psi(s) d s
$$


at $z \in[0,1]$. It is easy to see that this defines a nice function $\xi_{2}$ for any $\psi$ satisfying (96). Moreover,

$$
\xi_{2}^{\prime \prime}(z)=\frac{1}{I_{0}^{+}+I_{0}^{-}} \psi(z) .
$$

We now assume that $\xi_{2}$ is given by (98) where the choice of $\psi$ will be specified below. Note that the inequality (93) which must be satisfied by the function $\xi_{0}$ is rewritten as

$$
(1-z) \xi_{0}^{\prime \prime}(z)-(2-v) \xi_{0}^{\prime}(z) \leq \frac{1}{I_{0}^{+}+I_{0}^{-}} \chi(z) \psi(z) .
$$

We look for a nice function $\xi_{0}$ which satisfies the equation

$$
(1-z) \xi_{0}^{\prime \prime}(z)-(2-v) \xi_{0}^{\prime}(z)=\kappa(z) \chi(z) \frac{\psi(z)}{I_{0}^{+}+I_{0}^{-}}, \quad z \in[0,1]
$$

Here we write

$$
\kappa(z)= \begin{cases}\kappa_{+}, & \text {for } z \in[0, \zeta], \\ \kappa_{-}, & \text {for } z \in[\zeta, 1],\end{cases}
$$

for some constant $\kappa_{ \pm}$such that

$$
\kappa_{+}<1<\kappa_{-} .
$$

The integration of (100) gives

$$
\xi_{0}(z)=\left\{\begin{aligned}
& \frac{\kappa_{+} \chi_{+}}{\left(I_{0}^{+}+I_{0}^{-}\right)(1-v)} \\
& \times \int_{0}^{z} \psi(s)\left(\left(\frac{1-s}{1-z}\right)^{1-v}-1\right) d s, \quad \text { for } z \in[0, \zeta], \\
1+ & \frac{\kappa_{-} \chi_{-}}{\left(I_{0}^{+}+I_{0}^{-}\right)(1-v)} \\
& \times \int_{z}^{1} \psi(s)\left(1-\left(\frac{1-s}{1-z}\right)^{1-v}\right) d s, \quad \text { for } z \in[\zeta, 1] .
\end{aligned}\right.
$$

It is seen that $\xi_{0}(0)=0, \xi_{0}(1)=1$. We also have

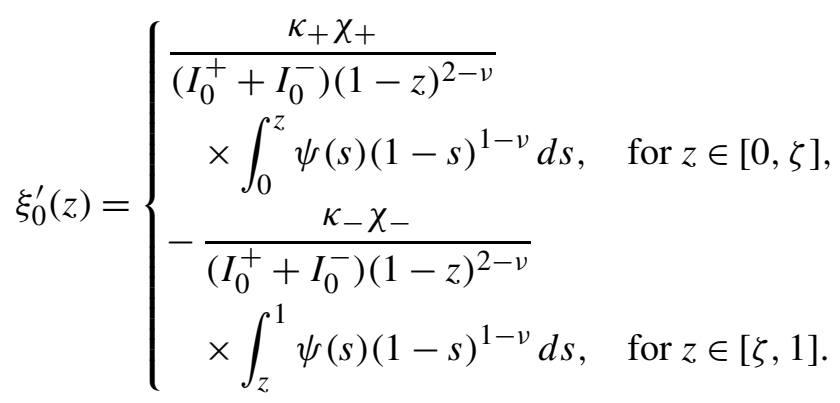


Thus, $\xi_{0}^{\prime}(z)>0$ at $z \in(0,1)$ and

$$
\xi_{0}^{\prime}(0)=0, \quad \xi_{0}^{\prime}(1)=-\frac{\kappa_{-} \chi_{-}}{\left(I_{0}^{+}+I_{0}^{-}\right)(2-v)} \psi(1)=0 .
$$

One can also check that

$$
\xi_{0}^{\prime \prime}(0)=\frac{\kappa_{+} \chi_{+}}{I_{0}^{+}+I_{0}^{-}} \psi(0)=0 \quad \text { and } \quad \xi_{0}^{\prime \prime}(1)=-\frac{\kappa_{-} \chi_{-}}{\left(I_{0}^{+}+I_{0}^{-}\right)(3-v)} \psi^{\prime}(1) .
$$

It follows that to have a nice function $\xi_{0}$ we must assume additionally that $\psi^{\prime}(1)=0$ and that the continuity conditions

$$
\xi_{0}(\zeta-0)=\xi_{0}(\zeta+0), \quad \xi_{0}^{\prime}(\zeta-0)=\xi_{0}^{\prime}(\zeta+0)
$$

are fulfilled [the continuity of the second derivative would then follow from (100) since $\psi(\zeta)=0$ by assumption]. By (103) and (104) the continuity conditions are written as

$$
\kappa_{+} \chi_{+} I_{v}^{+}=\kappa_{-} \chi_{-} I_{v}^{-}
$$

and

$$
1-\frac{\kappa_{-} \chi_{-}}{(1-v)\left(I_{0}^{+}+I_{0}^{-}\right)}=-\frac{\kappa_{+} \chi_{+}}{(1-v)\left(I_{0}^{+}+I_{0}^{-}\right)}
$$

[note that we took into account equality (96)]. This leads to the following formula:

$$
\kappa_{ \pm}=\frac{(1-v)}{\chi_{ \pm} I_{v}^{ \pm}} \frac{I_{0}^{+}+I_{0}^{-}}{\left(1 / I_{v}^{-}\right)-\left(1 / I_{v}^{+}\right)} .
$$

To fulfill (102) at a sufficiently small $v$, it is enough to have

$$
\frac{\chi_{-}}{I_{0}^{+}}<\frac{I_{0}^{+}+I_{0}^{-}}{I_{0}^{+}-I_{0}^{-}}<\frac{\chi_{+}}{I_{0}^{-}} .
$$

By (95), this will be satisfied if $I_{0}^{+}$is close enough to 1 and $I_{0}^{-}$is close enough to zero. To this aim, just take $\psi$ sufficiently closely approximating the sum of the delta-function near zero and the minus delta-function near 1 .

So, fixing the choice of a smooth function $\psi$ such that (106) and (96) were satisfied [along with the requirements $\psi(0)=0, \psi(1)=0, \psi^{\prime}(1)=0, \psi(\zeta)=0$ and $\psi(z)>0$ at $z \in(0, \zeta)$ and $\psi(z)<0$ at $z \in(\zeta, 1)]$, we obtain the nice functions $\xi_{2}$ and $\xi_{0}$ satisfying (100) and (98), respectively. By (102), this means that inequality (93) holds for these two functions.

It follows that the function $\xi_{1}$ recovered from (92) is also nice and satisfies (91). Hence, for such chosen functions $\xi_{1,2}$ the function $U^{(q+1)}$ given by (85) is a supersolution indeed at all $r \in\left[R_{1}, R_{q+1}\right]$.

As required, $U^{(q+1)}$ is constant at $r=R_{q+1}$ :

$$
U^{(q+1)}\left(R_{q+1}, \varphi\right)=u_{q}+1-C \delta_{q} \equiv u_{q+1} .
$$


Hence,

$$
u_{q}+1 \geq u_{q+1}
$$

and, by taking $\delta_{q}$ small, we may always ensure

$$
u_{q+1} \geq u_{q}+\frac{1}{2} \text {. }
$$

Therefore,

$$
q \geq u_{q} \geq \frac{q}{2}
$$

By construction,

$$
U^{(q+1)}(x) \leq u_{q+1}
$$

and

$$
U^{(q+1)}(x) \geq u_{q+1}-1 \quad \text { at } r \geq R_{q} .
$$

It follows, first, that the supersolution $U$ which we obtain in the limit of this procedure as $q \rightarrow+\infty$ grows to infinity:

$$
\lim _{r \uparrow \infty|x|=r} \inf _{|x|=r} U(x)=+\infty .
$$

On the other hand, this growth can be made arbitrarily slow: it is seen that $U(x) \leq q$ at $|x| \leq R_{q}$ but $R_{q}$ may be taken growing as fast as necessary.

\section{APPENDIX B}

A review of criticality theory. Let $L$ be as in (1). Then there exists a corresponding diffusion process $Y$ on $\mathbb{R}^{d}$ that solves the generalized martingale problem for $L$ on $\mathbb{R}^{d}$ (see [12], Chapter 1). The process lives on $\mathbb{R}^{d} \cup \Delta$ with $\Delta$ playing the role of a cemetery state. We denote by $\mathbb{P}_{x}$ and $\mathbb{E}_{x}$ the corresponding probabilities and expectations, and define the transition measure $p(t, x, d y)$ for $L+\beta$ by

$$
p(t, x, B)=\mathbb{E}_{x}\left(\exp \left(\int_{0}^{t} \beta\left(Y_{s}\right) d s\right) ; Y_{t} \in B\right),
$$

for measurable $B \subseteq \mathbb{R}^{d}$.

DEFINITION 35. If

$$
\int_{0}^{\infty} p(t, x, B) d t=\mathbb{E}_{x} \int_{0}^{\infty} \exp \left(\int_{0}^{t} \beta\left(Y_{s}\right) d s\right) \mathbb{1}_{B}\left(Y_{t}\right) d t<\infty,
$$

for all $x \in \mathbb{R}^{d}$ and all bounded $B \subset \mathbb{R}^{d}$, then

$$
G(x, d y)=\int_{0}^{\infty} p(t, x, d y) d t
$$

is called Green's measure for $L+\beta$ on $\mathbb{R}^{d}$. If the above condition fails, then Green's measure for $L+\beta$ on $\mathbb{R}^{d}$ is said not to exist. 
In the former case, $G(x, d y)$ possesses a density, $G(x, d y)=G(x, y) d y$, which is called Green's function for $L+\beta$ on $\mathbb{R}^{d}$.

For $\lambda \in \mathbb{R}$ define

$$
C_{L+\beta-\lambda}=\left\{u \in C^{2}:(L+\beta-\lambda) u=0 \text { and } u>0 \text { in } \mathbb{R}^{d}\right\} .
$$

The operator $L+\beta-\lambda$ on $\mathbb{R}^{d}$ is called subcritical if Green's function exists for $L+\beta-\lambda$ on $\mathbb{R}^{d}$; in this case $C_{L+\beta-\lambda} \neq \varnothing$. If Green's function does not exist for $L+\beta-\lambda$ on $\mathbb{R}^{d}$, but $C_{L+\beta-\lambda} \neq \varnothing$, then the operator $L+\beta-\lambda$ on $\mathbb{R}^{d}$ is called critical. In this case $C_{L+\beta-\lambda}$ is one-dimensional. The unique function (up to a constant multiple) in $C_{L+\beta-\lambda}$ is called the ground state of $L+\beta$ on $\mathbb{R}^{d}$. The formal adjoint of the operator $L+\beta-\lambda$ on $\mathbb{R}^{d}$ is also critical with ground state $\tilde{\phi}$. If furthermore $\phi \tilde{\phi} \in L^{1}\left(\mathbb{R}^{d}\right)$, we call $L+\beta-\lambda$ on $\mathbb{R}^{d}$ product-critical. (For $\phi=\tilde{\phi}$ this means that $\phi$ is an $L^{2}$-eigenfunction.) Finally, if $C_{L+\beta-\lambda}=\varnothing$, then $L+\beta-\lambda$ on $\mathbb{R}^{d}$ is called supercritical.

If $\beta \equiv 0$, then $L+\beta$ is not supercritical on $\mathbb{R}^{d}$ since the function $f \equiv 1$ satisfies $L f=0$ on $\mathbb{R}^{d}$. In this case $L+\beta=L$ is subcritical or critical on $\mathbb{R}^{d}$ according to whether the corresponding diffusion process $Y$ is transient or recurrent on $\mathbb{R}^{d}$. Product criticality in this case is equivalent to positive recurrence for $Y$. If $\beta \leq 0$ and $\beta \not 0$, then $L+\beta$ is subcritical on $\mathbb{R}^{d}$.

In terms of the solvability of inhomogeneous Dirichlet problems, subcriticality guarantees that the equation $(L+\beta) u=-f$ in $\mathbb{R}^{d}$ has a positive solution $u$ for every $0 \lessgtr f \in C_{c}^{\eta}$. (Here $C_{c}^{\eta}=C_{c} \cap C^{\eta}$.) If subcriticality does not hold, then there are no positive solutions for any $0 \lesseqgtr f \in C_{c}^{\eta}$.

One of the two following possibilities holds:

1. There exists a number $\lambda_{c} \in \mathbb{R}$ such that $L-\lambda$ on $\mathbb{R}^{d}$ is subcritical for $\lambda>\lambda_{c}$, supercritical for $\lambda<\lambda_{c}$, and either subcritical or critical for $\lambda=\lambda_{c}$.

2. $L-\lambda$ on $\mathbb{R}^{d}$ is supercritical for all $\lambda \in \mathbb{R}$, in which case we define $\lambda_{c}=\infty$.

Definition 36. The number $\lambda_{c} \in(-\infty, \infty]$ is called the generalized principal eigenvalue for $L$ on $\mathbb{R}^{d}$.

Note that $\lambda_{c}=\inf \left\{\lambda \in \mathbb{R}: C_{L+\beta-\lambda} \neq \varnothing\right\}$. Also, if $\beta$ is bounded from above, then case 1 holds.

If $L+\beta$ is symmetric with respect to a reference measure $\rho d x$, then $\lambda_{c}$ equals the supremum of the spectrum of the self-adjoint operator on $L^{2}\left(\mathbb{R}^{d}, \rho d x\right)$ obtained from $L+\beta$ via the Friedrichs extension theorem.

Let $h \in C^{2, \eta}$ satisfy $h>0$ in $\mathbb{R}^{d}$. The operator $(L+\beta)^{h}$ defined by

$$
(L+\beta)^{h} f=\frac{1}{h}(L+\beta)(h f)
$$

is called the $h$-transform of the operator $L+\beta$. Written out explicitly, one has

$$
(L+\beta)^{h} f=L+a \frac{\nabla h}{h} \cdot \nabla+\beta+\frac{L h}{h} .
$$


All the properties defined above are invariant under $h$-transforms.

For further elaboration and proofs see [12], Chapter 4.

Acknowledgments. We are grateful to M. Effendiev, K. Fleischmann, J. Gärtner, R. Pinsky, K. Schneider, A. Winter and M. Wolfrum for helpful discussions. The hospitality of the Weierstrass Institute is gratefully acknowledged by the first author.

\section{REFERENCES}

[1] Athreya, K. B. and Ney, P. E. (1972). Branching Processes. Springer, Berlin.

[2] Bramson, M., CoX, J. T. and Greven, A. (1993). Ergodicity of critical spatial branching processes in low dimensions. Ann. Probab. 21 1946-1957.

[3] Dawson, D. A. (1993). Measure-valued Markov processes. École d'Été de Probabilités de Saint Flour XXI. Lecture Notes in Math. 1541 1-260. Springer, Berlin.

[4] Dynkin, E. B. (1991). Branching particle systems and superprocesses. Ann. Probab. $191157-$ 1194.

[5] Dynkin, E. B. (1993). Superprocesses and partial differential equations. Ann. Probab. 21 $1185-1262$.

[6] Effendiev, M. A. and ZeliK, S. V. (2001). The attractor for a nonlinear reaction-diffusion system in the unbounded domain. Comm. Pure Appl. Math. 54 625-688.

[7] Engländer, J. and Fleischmann, K. (2000). Extinction properties of super-Brownian motions with additional spatially dependent mass production. Stochastic Process. Appl. $8837-58$.

[8] Engländer, J. and Pinsky, R. G. (1999). On the construction and support properties of measure-valued diffusions on $D \subset R^{d}$ with spatially dependent branching. Ann. Probab. 27 684-730.

[9] Engländer, J. and Winter, A. (2002). Strong law of large numbers for locally surviving superdiffusions. Unpublished manuscript.

[10] Feller, W. (1971). An Introduction to Probability Theory and its Applications 2, 2nd ed. Wiley, New York.

[11] Friedman, A. (1964). Partial Differential Equations of Parabolic Type. Prentice Hall, New York.

[12] Pinsky, R. G. (1995). Positive Harmonic Functions and Diffusion. Cambridge Univ. Press.

[13] PINSKY, R. G. (1996). Transience, recurrence and local extinction properties of the support for supercritical finite measure-valued diffusions. Ann. Probab. 24 237-267.

[14] PInsky, R. G. (2001). Invariant probability distributions for measure-valued diffusions. Ann. Probab. 29 1476-1514.

[15] Shilnikov, L., Shilnikov, A., Turaev, D. and Chua, L. (1998). Methods of Qualitative Theory in Nonlinear Dynamics, Part I. World Scientific, Singapore.

[16] Walther, H.-O. (1987). Inclination lemmas with dominated convergence. Z. Angew. Math. Phys. 28 327-337.

EURANDOM

P.O. BOX 513

NL-5600 MB EINDHOVEN

THE NETHERLANDS

E-MAIL: jengland@euridice.tue.nl
WEIERSTRASS INSTITUTE FOR APPLIED ANALYSIS AND STOCHASTICS

MOHRENSTRASSE 39

D-10117 BERLIN

GERMANY

E-MAIL: turaev@wias-berlin.de 